\title{
Random walks derived from billiards
}

\author{
RENATO FERES
}

To Anatoly Katok, on his 60th birthday

\begin{abstract}
We introduce a class of random dynamical systems derived from billiard maps, which we call random billiards, and study certain random walks on the real line obtained from them. The interplay between the billiard geometry and the stochastic properties of the random billiard is investigated. Our main results are concerned with the spectrum of the random billiard's Markov operator. We also describe some basic properties of diffusion limits under appropriate scaling.
\end{abstract}

\section{Introduction}

This work is motivated by the following problem about gas kinetics. Suppose that a short pulse of inert gas at very low pressure is released from a point inside a long but finite cylindrical channel. The time at which gas molecules escape the channel through its open ends is then measured by some device such as a mass spectrometer. The inner surface of the cylinder is not perfectly flat due to its molecular structure, imagined as a periodic relief. It is not altogether unreasonable to think that the interaction between the fast moving (inert) gas molecules and the surface is essentially elastic, and that any thermal effects can be disregarded on first approximation. (See $[\mathrm{ACM}]$ for a more detailed physical justification of this assumption.) We thus think of the gas-surface interaction as billiard-like. (M. Knudsen, in his classical theoretical and experimental studies on the kinetic theory of gases begun around 1907, used a tennis ball metaphor [Kn, p. 26].) The assumption of low pressure simply means that the collisions among gas molecules are in sufficiently small numbers to be disregarded and only collisions between gas molecules and the channel inner surface are taken into account. The problem is now this: from the time of escape data, possibly 
for a range of values of channel length, we wish to extract information about the microgeometry of the channel surface.

A mathematical formulation of this problem is proposed below in terms of random dynamical systems derived from deterministic billiards. For each choice of billiard geometry, the interaction between gas molecules and channel inner surface is encoded in a Markov (scattering) operator, $P$. The study of $P$ is the main focus of the paper, but we also consider to a lesser extent the random flight inside the channel derived from $P$, and make a number of general remarks about the asymptotic behavior of the time of escape for long channels. More details about the relationship between geometric properties of the billiard cells and diffusion characteristics of (limits of) the random walk will be explored in a future paper. (For a numerical study of this relationship, see [FY2].) The definitions given here were introduced in essentially nonmathematical form in our [FY1].
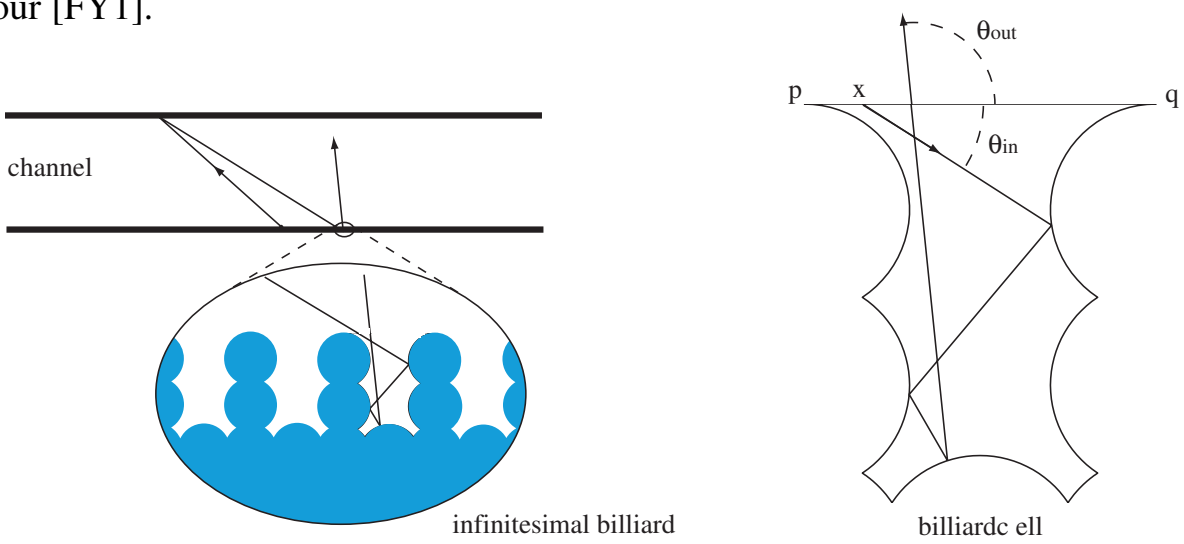

Figure 1. Channel with microgeometry, showing the initial steps of a billiard particle flight (left) and the trajectory inside a billiard cell (right). When the billiard cell is regarded as infinitesimal in the sense defined in the text, the initial point $x$ on the open side $\overline{p q}$ becomes a random variable uniformly distributed over the interval from $p$ to $q$. The angle $\theta_{\text {out }}$ then becomes a random function of $\theta_{\text {in }}$. The transition probabilities of the events $\theta_{\text {in }} \mapsto \theta_{\text {out }}$ are described by a Markov operator $P$ canonically specified by the cell's shape.

The wished-for general theory, of which the present paper is only the first step, can be seen in a nutshell with the help of a simple example. (The mainly numerical study [FY2] shows a more detailed overview, with many more examples. See also Section 7.) Before describing the example, let us define the time-of-escape function, $f(x)$, a little more precisely. Consider the quantity $\tau(L, r, v)$, defined as the mean time of escape of a pulse of billiard particles released from the middle point of a channel of length $2 L$ and radius $r$, where 
$v$ is the scalar velocity, assumed equal for all particles. It is convenient to work with a dimensionless quantity $f(x)$, where $x=L / r$, given by

$$
\tau(L, r, v)=\frac{r}{v} f(x) .
$$

That $\tau$ indeed has such a functional form is due to the elementary observation that for any positive number $c$, the mean time of escape satisfies the relations $\tau(c L, c r, c v)=\tau(L, r, v)$ and $\tau(L, r, c v)=\tau(L, r, v) / c$. Notice that $f(x)$ depends exclusively on the microgeometry of the channel surface encoded by $P$. The central problem of the theory is to extract information about this microgeometry from $f(x)$.

Let the microgeometry be that of Figure 2. There are two geometric parameters: $h$ and $b$. It will be clear from the definition of $P$ that it only depends on the shape of the billiard cell up to homothety. So the only geometric parameter we can hope to recover from this time-of-escape experiment is the ratio $b / h$.
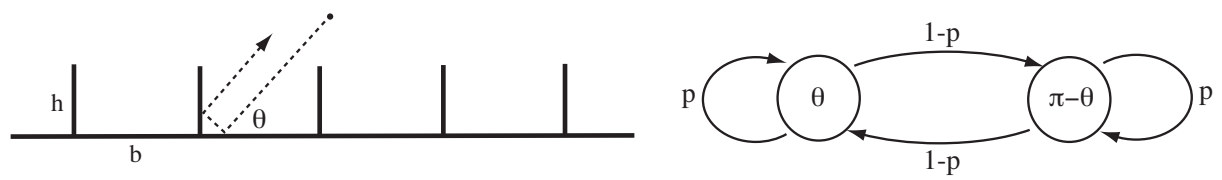

Figure 2. Transition probabilities for this "fence microgeometry" are given by the above Markov diagram. Standard random walk on $\mathbb{R}(p=1 / 2)$ corresponds to the projection of the random flight in a channel of radius $r=1 / 2, \theta=\pi / 4$, and $b / h=4$.

The transition probabilities, represented by $p$ and $1-p$ in the diagram on the right-hand side of Figure 2, are given as follows. Let $k \in \mathbb{Z}$ denote the integer part of $2 h / b \tan \theta$, and $s \in[0,1)$ the fractional part, so that

$$
\frac{2 h}{b \tan \theta}=k+s \text {. }
$$

Then, if $k$ is even, $p=1-s$, and if $k$ is odd, $p=s$. This is easily obtained by inspecting the unfolding of the billiard cell as shown in Figure 3.

The Markov operator completely determines the microgeometry, up to scale, for this (one-parameter) family of geometries. In other words, the quantity $h / b$ is known if we know the transition probabilities for all values of $\theta$. The standard random walk on a line (which has probability $1 / 2$ of jumping either forward or backward by a fixed length) corresponds to the horizontal projection of a random flight in a 2-dimensional channel of radius $r=1 / 2, v=1$, and box dimensions $\theta=\pi / 4$, and $b / h=4$.

It can also be shown that the parameter $b / h$ is recovered from the time-ofescape function $f(x)$. For concreteness, let us suppose that the initial direction 


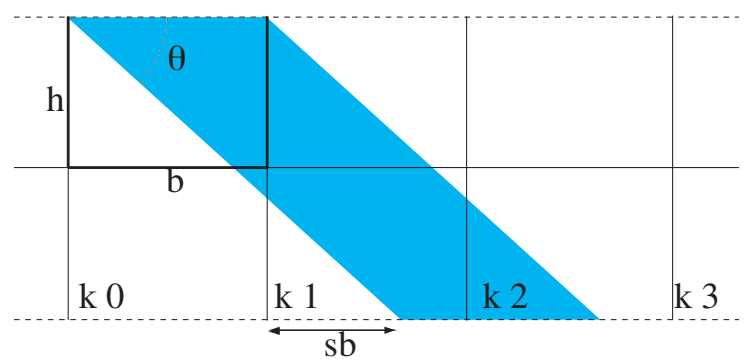

Figure 3. Unfolding of a billiard cell of Figure 2, used to determine the transition probabilities.

is given by the angle $\theta=\pi / 4$ and the lengths of the base, $b$, and height, $h$, of the rectangular cell satisfy $b>2 h$. Then (see Section 7), for large values of $x$ (i.e., large ratio $L / r$ ), we have the asymptotic expression

$$
\frac{f(x)}{x^{2}} \sim \frac{1}{\sqrt{2}}\left(\frac{b}{2 h}-1\right)^{-1} .
$$

Therefore, the single geometric parameter of this family of microgeometries, namely the ratio $b / h$, can be recovered from $f(x)$. This follows from a central limit theorem for Markov chains, which can also be used to show that for long channels the probability density, $u(t, z)$, of finding a billiard particle at time $t$ and position $z$ along the axis of the channel satisfies a standard diffusion equation

$$
\frac{\partial u}{\partial t}=D \frac{\partial^{2} u}{\partial z^{2}}
$$

where $D=(1 / \sqrt{2})(b / 2 h-1) r v$ is the diffusion constant, $r$ is the channel radius, and $v$ is the constant scalar velocity of the billiard particles.

The main problem is thus to understand how much geometric information about the billiard cell is contained in $f(x)$. Another, relatively simpler (though not simple), problem is to understand how much geometric information is contained in the spectrum of the operator $P$. As will be seen later in the paper, $P$ will have discrete real spectrum for a large class of billiard geometries. We should note that the above example is not entirely representative of most microgeometries in some important ways. Most importantly, the correct asymptotic expression of $f(x)$ for large $x$ will typically be $D^{-1} x^{2} / \ln x$, rather than $D^{-1} x^{2}$, where $D$ is a diffusion constant. This is briefly discussed in Section 7 .

The paper is organized as follows. The first section introduces the idea of channel microgeometry and the way a point particle interacts with it. This naturally leads to the definition of a random billiard map, generating a random dynamical system on a set $V$. Here $V$ stands for the interval $[0, \pi]$ in the 
two-dimensional version of the problem, or the unit hemisphere in the threedimensional version. The microgeometry specifies a Markov chain with state space $V$ whose transition probabilities operator, $P$, replaces the ordinary reflection law of deterministic billiards. The Markov chain gives at each moment in time the velocity component of a random flight inside the channel.

The general properties of the random billiard Markov chain are then investigated. It is shown that this random process has a canonical stationary measure, $\mu$, obtained from the Liouville measure of the associated deterministic billiard. A simple ergodicity criterion for $\mu$ is provided.

It is shown next that $P$ is a bounded self-adjoint operator on $L^{2}(V, \mu)$. Selfadjointness is a consequence of time reversibility of the deterministic system, which also implies reversibility of the Markov chain. Under more stringent assumptions (essentially that the underlying deterministic billiard is a dispersing, or Sinai, billiard) it is shown that $P$ is a Hilbert-Schmidt operator. This is one of the central results of the paper.

We then turn to the associated random flight. It is shown by means of an appropriate central limit theorem how the random flight gives rise to Brownian motion, with variance that depends on the spectrum of $P$, hence on the microgeometry of the channel. This is accomplished most easily in cases when the stationary measure is nonergodic and does not contain the direction of the axis of the channel in its support. The general (ergodic) case, which we consider only briefly, mainly through one example, requires a more careful analysis as the jumps of the random walk have infinite variance. The probability distribution of these jumps is, nevertheless, in the domain of attraction of a normal distribution and ordinary diffusion can still be obtained under an appropriate scaling limit. Explicit values for the diffusion constant are derived for a few simple examples. Finally, explicit formulas for the mean time and mean number of collisions inside a billiard cell are given in terms of parameters associated to the shape of the cell.

Acknowledgment. This paper has benefited from conversations with M. Nicol and D. Szász. I wish to express my thanks to them.

\section{Main definitions}

The central concepts of the paper-the definitions of a random billiard, the microgeometry of a channel, the associated Markov operator and random flight in a channel—are introduced here.

2.1. Reflection off a wall with infinitesimal structure. A surface (or curve) microgeometry, and how a point particle interacts with it, is the first idea that needs to be explained. The precise description of the particle-surface interaction 
will involve the notion of a random billiard, defined in the next subsection. Although not logically needed, the following remark should provide a conceptual justification for the definitions. The key point to clarify is how to define the reflection law for a billiard particle bouncing off a wall that has periodic geometric features at an infinitesimal scale.

Consider a family of piecewise differentiable surfaces, or curves, $S_{a}$, which approximates (in Hausdorff metric) another piecewise differentiable surface $S$ as indicated by Figure 4. Each $S_{a}$ can be thought of as superposing to $S$ a periodic geometry scaled down in size, with scale parameter $a$, and slightly deformed to account for the curvature of $S$. A procedure that yields a welldefined limit for the billiard reflection as $a \rightarrow 0$ is the following. As a first step, we replace the incoming velocity $v$ with a random variable $V_{\varepsilon}$ whose probability distribution has smooth density and is sharply concentrated around $v$, approaching $v$ as $\varepsilon \rightarrow 0$. The distribution of reflected velocities is then obtained, resulting in a random variable with probability measure $\mu_{a, \varepsilon}^{v}$. The reflection law for the incident velocity, $v$, is now defined by the limit (in the weak* topology)

$$
\mu_{v}:=\lim _{\varepsilon \rightarrow 0} \lim _{a \rightarrow 0} \mu_{a, \varepsilon}^{v}
$$

It can be shown that the limit exits and the resulting measure is uniquely determined by the scaled down geometry. It is not difficult to obtain, using elementary facts about oscillatory integrals, that the limit has a very explicit form. In dimension two (writing $\mu_{v}=\mu_{\theta}$ ) it is given by

$$
\mu_{\theta}(h)=\int_{0}^{1} h\left(\Psi_{x}(\theta)\right) d x
$$

where $\theta \in(0, \pi)$ is the incidence angle measured with respect to the tangent line to $S$ at the point of collision, $x \in[0,1]$ parametrizes a point on the open

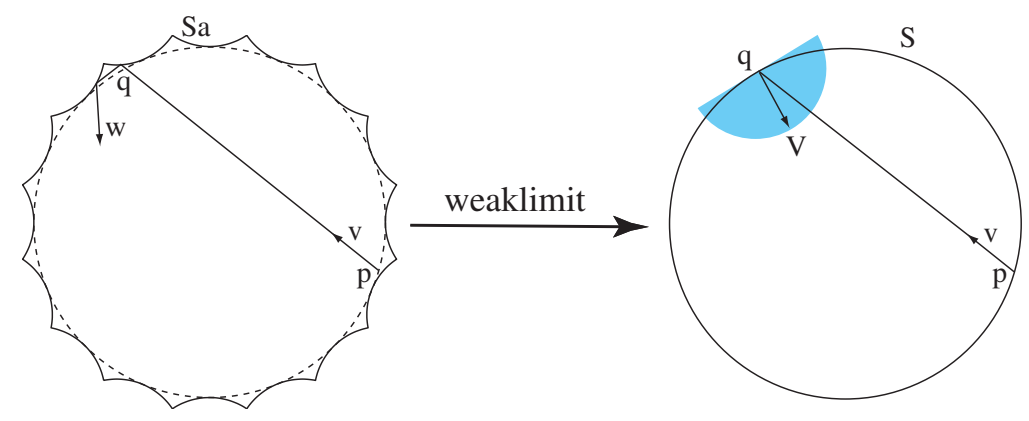

Figure 4. $S$ is fixed and $S_{a}$ varies so that bumps scale down to zero in size. As sets, $S_{a} \rightarrow S$, but reflection law becomes probabilistic, represented by a scattering operator. 
side of one of the "microscopic" indentations of $S_{a}$ inside of which the particle executes a billiard ball motion (see Figure 4), $\Psi_{x}(\theta)$ is the angle that the particle makes with $S$ as it leaves the indentation, and $\mu_{\theta}(h)$ denotes the integral of an arbitrary continuous function $h$ on $[0, \pi]$ with measure $\mu_{\theta}$. (See [FY1] for more details about the above limit.) In dimension $3, d x$ is Lebesgue measure over the unit square.

The key observation here is that the direction of reflection for an incoming velocity $v$ should be regarded as a random variable. Its probability law, $\mu_{v}$, is obtained by assuming that the point on the open side of the indentation through which the particle passes is a random variable uniformly distributed over the area (or length) of that open side. The indentations are the billiard cells of the random billiard, as defined next.

2.2. Random billiards. The billiard table with an open side that goes into the definition of a random billiard should be thought as representing the individual indentations of the surface $S_{a}$ for a very small $a$. Consider an ordinary billiard system consisting of a billiard table $B$. The boundary of $B$ is the union of a finite number of smooth curves, called the sides of the table. One of the sides is distinguished, and for our purposes it will always be a segment of line (or the 2-torus in dimension 3; see below), which will be called the open side. This is the segment $p q$ of the billiard cell on the right-hand side of Figure 1.

Choose a number $s \in[0,1]$ at random with uniform probability and set the initial point of the billiard trajectory to be $x=p+s(q-p)$. Denote by $\Psi_{x}:[0, \pi] \rightarrow[0, \pi]$ the angle component of the first return map of the billiard trajectory back to the open side. Referring to Figure 1 (right-hand side), we have $\theta_{\text {out }}=\Psi_{x}\left(\theta_{\text {in }}\right)$. We often identify the open side with the interval $[0,1]$. The first return map to $p q$ of the ordinary billiard is a map $T$ from the rectangle $[0,1] \times[0, \pi]$ to itself such that $T(x, \theta)=\left(y, \Psi_{x}(\theta)\right)$. The random billiard is then the random dynamical system $\left(\left\{\Psi_{x}: x \in[0,1]\right\}, \mathscr{B}, \lambda\right)$, where $\lambda$ is Lebesgue measure on the unit interval and $\mathscr{B}$ is the Borel $\sigma$-algebra.

Figure 5 provides one interpretation for the iteration of a random billiard map. The table $B$ is doubled over its flat side; a trajectory of the random billiard is

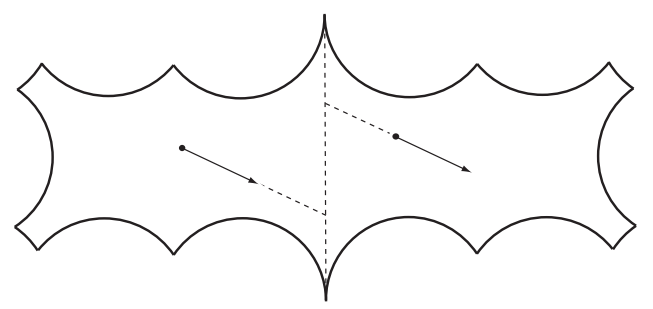

Figure 5. Doubling of billiard table with a random map. 
an ordinary billiard trajectory until it crosses the separation line between the two copies of $B$ (the dashed line in Figure 5), at which moment it jumps to a randomly chosen point on the line, keeping the velocity unchanged.

As an example of random billiard map, consider the (doubled up) triangular table shown in Figure 6. Let $\alpha<\pi / 6$. Define maps $T_{i}:[0, \pi] \rightarrow \mathbb{R}$ by

$$
\begin{aligned}
& T_{1}(\theta)=\theta+2 \alpha \\
& T_{2}(\theta)=-\theta+2 \pi-4 \alpha \\
& T_{3}(\theta)=\theta-2 \alpha \\
& T_{4}(\theta)=-\theta+4 \alpha .
\end{aligned}
$$

The random billiard map $T:[0, \pi] \rightarrow[0, \pi]$ is given by $T(\theta)=T_{i}(\theta)$ with probability $p_{i}(\theta)$. To specify $p_{i}$, first define the function

$$
u_{\alpha}(\theta)=\frac{1}{2}\left(1+\frac{\tan \alpha}{\tan \theta}\right) \text {. }
$$

Now define

$$
\begin{aligned}
& p_{1}(\theta)= \begin{cases}1 & \theta \in[0, \alpha) \\
u_{\alpha}(\theta) & \theta \in[\alpha, \pi-3 \alpha) \\
2 \cos (2 \alpha) u_{2 \alpha}(\theta) & \theta \in[\pi-3 \alpha, \pi-2 \alpha) \\
0 & \theta \in[\pi-2 \alpha, \pi]\end{cases} \\
& p_{2}(\theta)= \begin{cases}0 & \theta \in[0, \pi-3 \alpha) \\
u_{\alpha}(\theta)-2 \cos (2 \alpha) u_{2 \alpha}(\theta) & \theta \in[\pi-3 \alpha, \pi-2 \alpha) \\
u_{\alpha}(\theta) & \theta \in[\pi-2 \alpha, \pi-\alpha) \\
0 & \theta \in[\pi-\alpha, \pi]\end{cases} \\
& p_{3}(\theta)= \begin{cases}0 & \theta \in[0,2 \alpha) \\
2 \cos (2 \alpha) u_{2 \alpha}(-\theta) & \theta \in[2 \alpha, 3 \alpha) \\
u_{\alpha}(-\theta) & \theta \in[3 \alpha, \pi-\alpha) \\
1 & \theta \in[\pi-\alpha, \pi]\end{cases} \\
& p_{4}(\theta)= \begin{cases}0 & \theta \in[0, \alpha) \\
u_{\alpha}(-\theta) & \theta \in[\alpha, 2 \alpha) \\
u_{\alpha}(-\theta)-2 \cos (2 \alpha) u_{2 \alpha}(-\theta) & \theta \in[2 \alpha, 3 \alpha) \\
0 & \theta \in[3 \alpha, \pi] .\end{cases}
\end{aligned}
$$

Figure 6 shows the graph of the random map $T$.

If $\alpha=p \pi / q$, for $p, q$ positive integers, the maps $T_{i}$ generate a dihedral group, $D_{m}$ (of order $2 m$ ), where $m=q$ if $q$ is odd, and $m=q / 2$ if $q$ is even. The random dynamical system can be regarded as a random walk on an orbit $D_{m} \cdot \theta$, for $\theta \in[0, \pi]$. The generators of the random walk are $T_{1}, T_{2}, T_{3}=T_{1}^{m-1}, T_{4}=$ $T_{2} \circ T_{1}^{m-4}$, chosen with probabilities $p_{i}\left(\theta^{\prime}\right)$, for $\theta^{\prime}$ on the orbit of $\theta$. Notice that any pair $(\mathscr{P}, s)$, where $\mathscr{P}$ is a rational polygon in the plane (specified up to 


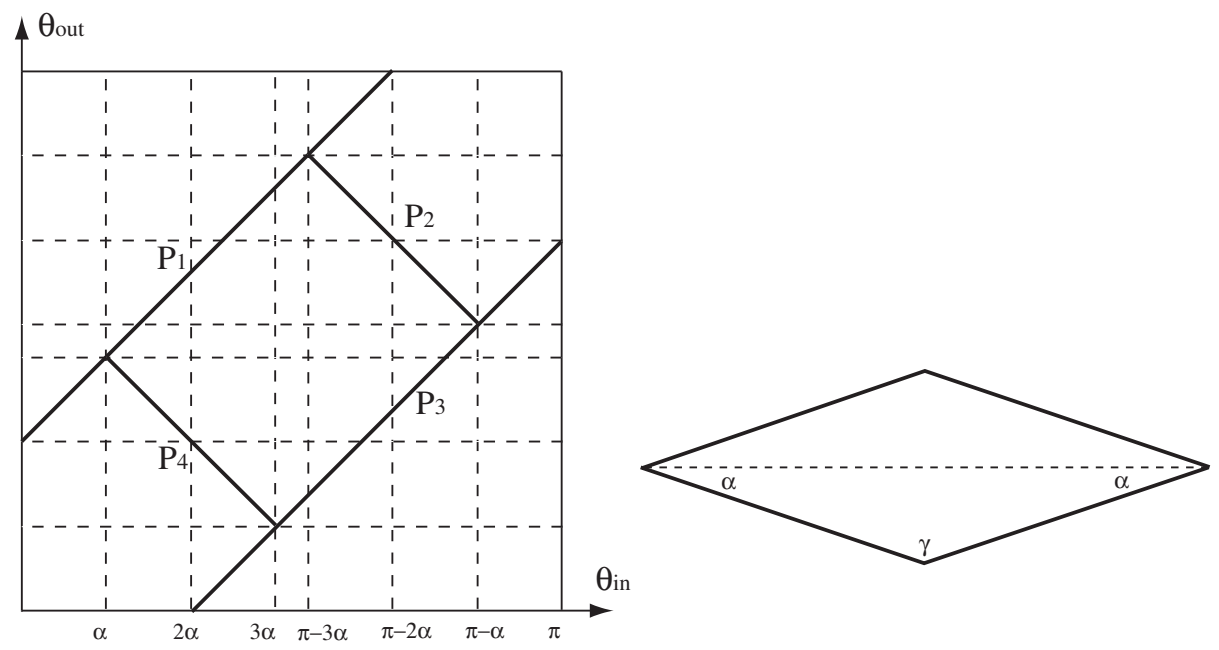

Figure 6. Random billiard map (left) for the shallow triangular table (lower half of the figure on the right).

a similarity transformation) and $s$ is a choice of side of $\mathscr{P}$, determines such a position dependent random walk on a dihedral group.

2.3. Dimension 3. In dimension 3 , the boundary surface of the billiard table will be a piecewise smooth surface without boundary, contained in $\mathbb{T}^{2} \times[0, a]$, that separates the top and bottom tori; that is, any geodesic segment in $\mathbb{T}^{2} \times[0, a]$ that starts in $\mathbb{T}^{2} \times\{a\}$ and ends in $\mathbb{T}^{2} \times\{0\}$ must intersect the surface. The top torus, denoted simply by $\mathbb{T}^{2}$, assumes the same role as the distinguished side of the two dimensional billiard. A trajectory of the three dimensional open billiard might look like the one shown in Figure 7. Incoming and outgoing velocities are parametrized by the upper half (unit) hemisphere,

$S^{+}:=\left\{v=\left(v_{1}, v_{2}, v_{3}\right): v_{3}>0,|v|=1\right\}$.

The random dynamical system is now a random map on $S^{+}$, denoted $\Psi_{x}: S^{+} \rightarrow S^{+}$, for $x \in \mathbb{T}^{2}$. The torus is given the normalized Lebesgue measure. Notice that any choice of $a$ (greater than the height of the

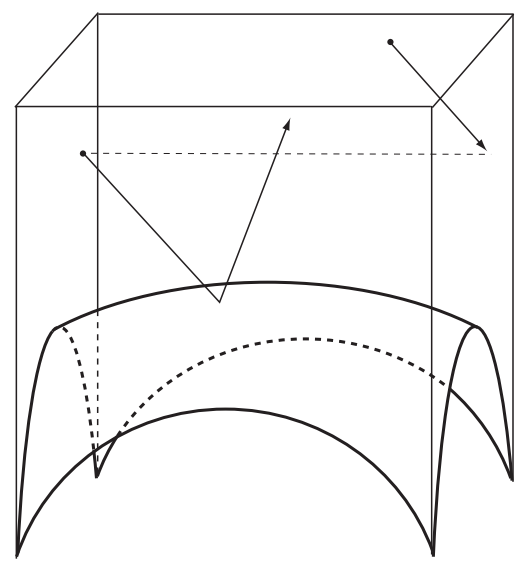

Figure 7. 3-D billiard cell. surface itself) will produce the same random dynamical system. 
The random billiard can be expressed as an ordinary (deterministic) dynamical system on an extended state space, as follows. In dimension 2, let $\Omega=[0,1]^{\mathbb{N}}$ and $v=\lambda^{\mathbb{N}}$, where $\lambda$ is the Lebesgue measure on the unit interval. Define the shift map $\sigma: \Omega \rightarrow \Omega$ by $\sigma(\omega)_{i}:=\omega_{i+1}$ and $\Psi_{\omega}:=\Psi_{\omega_{1}}$. This gives a map $\Psi: \Omega \times[0, \pi] \rightarrow \Omega \times[0, \pi]$. The $n$-th iterate of $\Psi$ is

$$
\Psi^{n}(\omega, \theta)=\left(\Psi_{\sigma^{n}(\omega)} \circ \cdots \circ \Psi_{\sigma(\omega)} \circ \Psi_{\omega}\right)(\theta) .
$$

The three-dimensional case is similarly defined.

We summarize here some notation about billiards that will be used consistently throughout the paper. Let $(X, \mathscr{B}, \lambda)$ denote the measure space $[0,1]$ or $\mathbb{T}^{2}$ with the normalized Lebesgue measure and the Borel $\sigma$-algebra. Let $V$ denote, respectively, $[0, \pi]$ or $S^{+}$, and $\Psi_{x}: V \rightarrow V$, the random billiard maps. At times it will be convenient to write $F_{v}(x):=\Psi_{x}(v)$. In what follows, $\mu$ will denote (unless explicitly stated otherwise) the probability measure on $V$ defined by $d \mu(\theta)=\frac{1}{2} \sin \theta d \theta$ if $V=[0, \pi]$, and $d \mu(v)=v \cdot n d A(v)$, for $V=S^{+}$, where $d A$ is the area element of $S^{+}$. In spherical coordinates, $d \mu(\theta, \phi)=(1 / 2 \pi) \sin (2 \theta) d \theta d \phi$, for $0 \leq \theta \leq \pi / 2$ and $0 \leq \phi \leq 2 \pi$.

Let $E=X \times V$ denote the phase space of the open side of the (deterministic) billiard table, both in dimension 2 and dimension 3 . The projection to second component will be written $\pi_{2}: E \rightarrow V$. The Liouville measure of the deterministic billiard gives, after restriction and normalization, the measure $v=\lambda \otimes \mu$ on $E$. Notice that $v$ is invariant under the first return map $T: E \rightarrow E$.

The random system can also be studied as a Markov chain, which is the point of view we mostly take. This will be described shortly, after we explain the connection between the random billiard and random flights in a channel. For detailed definitions and basic properties about random dynamical systems, we refer the reader to $[\mathrm{Ar}]$ and $[\mathrm{Re}]$.

2.4. Channels with microgeometry and random flights. By a channel we mean either a pair of parallel lines (dimension 2) or a cylinder (dimension 3). It may be of finite or infinite length. A channel with microgeometry is a channel with a choice of random billiard. In dimension 3 , we could more generally define a surface with microgeometry to be a triple $(S, \xi, \Psi)$, where $S$ is a piecewise smooth embedded surface in $\mathbb{R}^{3}, \xi$ is a piecewise smooth orthonormal framing of $S$, and $\Psi$ is a random billiard. For cylindrical channels, the framing is always the one represented by the vectors $e_{1}, e_{2}, n$ of Figure 8 . The normal vector, $n$, is taken so as to point into the region enclosed by the surface and $e_{2}$ is parallel to the axis of the cylinder.

To a channel with microgeometry it is associated a random flight in the obvious way: a particle with a given initial velocity moves along a straight line in uniform motion until it hits a point on the channel surface. It then reflects at an 
angle specified by the random billiard. Vectors at the point of collision are identified with vectors at the open side of a billiard cell by means of the orthonormal frame. In other words, we assume that the random billiard is "attached" to the frame in this sense: defining $C:\left(v_{1}, v_{2}, v_{3}\right) \mapsto\left(v_{1}, v_{2},-v_{3}\right)$, a particle that hits the surface at $p$ with velocity $v=v_{1} e_{1}(p)+v_{2} e_{2}(p)+v_{3} n(p)$ follows the direction specified by $w=w_{1} e_{1}(p)+w_{2} e_{2}(p)+w_{3} n(p)$ after reflection, where $\left(w_{1}, w_{2}, w_{3}\right)=C \Psi_{x}\left(v_{1}, v_{2}, v_{3}\right)$, for a randomly chosen $x$.

Geometric parameters of the channel (its radius and length) are not commensurate with those of the billiard cell. Consequently, any geometric characteristic of the cell that can affect the behavior of the random flight must be invariant under homothety, such as length ratios and angles.

It is, perhaps, not entirely obvious that iterations of the random billiard indeed correspond to the velocity process of the random flight in a cylindrical channel. This is because, in principle, one would need to apply a random rotation to $\Psi_{x}(v)$ after each collision, to account for the fact that the moving frame $\xi$ rotates from a point on the channel to the next. To clarify this point, we first suppose that the channel is an unspecified orientable differentiable surface embedded in $\mathbb{R}^{3}$, with a given framing $\xi=\left(e_{1}, e_{2}, n\right)$. Denote by $p\left(\sigma^{n}(\omega)\right), \omega \in \Omega$, the point of $n$-th collision with the surface for a random trajectory. Define $I_{p}:\left(v_{1}, v_{2}, v_{3}\right) \mapsto$ $v_{1} e_{1}+v_{2} e_{2}+v_{3} n$ and write

$$
R\left(\sigma^{n}(\omega)\right):=\left(I_{p\left(\sigma^{n+1}(\omega)\right)}\right)^{-1} \circ I_{p\left(\sigma^{n}(\omega)\right)} \circ C .
$$

The velocity after $n$ collisions is given by

$$
v \mapsto\left(I_{p\left(\sigma^{n} \omega\right)}\right)^{-1} \circ \Psi_{\sigma^{n}(\omega)} \circ R\left(\sigma^{n-1}(\omega)\right) \circ \Psi_{\sigma^{n-1}(\omega)^{\circ}} \cdots \circ \Psi_{\sigma(\omega)} \circ R(\omega) \circ \Psi_{\omega} \circ I_{p(\omega)} v .
$$

If the surface is a cylinder and the moving frame is chosen as indicated in Figure 8, then an elementary geometric argument shows that the orthogonal transformations $R(\omega)$ are equal to the identity matrix. Therefore, the random dynamical system defined by $\left\{\Psi_{x}: x \in \mathbb{T}^{2}\right\}$ actually describes the changing velocity of a particle in a random flight inside the cylinder as claimed.

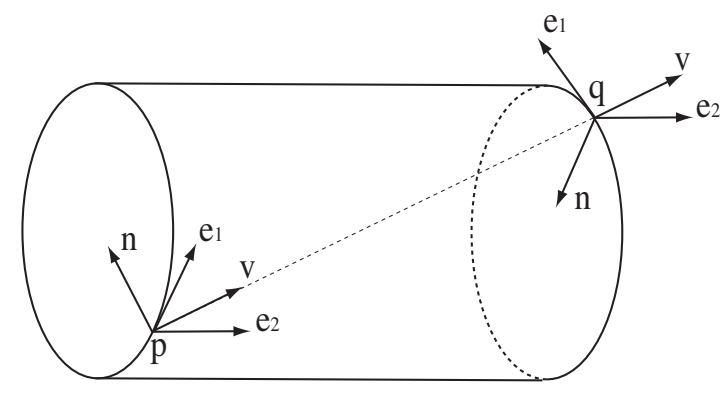

Figure 8. Standard frame over cylindrical surface. 
2.5. The associated Markov chain. Define a Markov chain with state space $V$ and transition probabilities

$$
P(A \mid v):=\left(F_{v}\right)_{*} \lambda(A):=\lambda\left(F_{v}^{-1}(A)\right)=\lambda^{\mathbb{N}}\left(\left\{\omega \in \Omega: \Psi_{\omega}(v) \in A\right\}\right) .
$$

The conditional expectation of a function $f$, given $v$, is

$$
\mathbb{E}[f \mid v]=\int_{X} f\left(F_{v}(x)\right) d \lambda(x) .
$$

The transition probability $P(A \mid v)$ should be interpreted as the probability that a particle will reflect with a velocity in $A$ given that the pre-collision velocity is $v$. More generally, define $P_{k}(A \mid v)=\lambda^{\mathbb{N}}\left(\left\{\omega \in \Omega: \Psi_{\omega}^{k}(v) \in A\right\}\right)$, for $k \in \mathbb{N}$. These $k$-step transition probabilities define a homogeneous Markov chain (see [Ar]). We have, in particular, the Chapman-Kolmogorov equation

$$
P_{k+l}(A \mid v)=\int_{V} P_{k}\left(A \mid v^{\prime}\right) P_{l}\left(d v^{\prime} \mid v\right),
$$

for $k, l \in \mathbb{N}$.

If $\mu$ is a probability measure on $V$ (not necessarily, for the moment, the velocity component of the Liouville measure), then there exists a unique probability measure, $P_{\mu}$, on the space $\Omega_{V}=V^{\mathbb{N}}$ with the product $\sigma$-algebra such that the coordinate functions, $\pi_{k}: \Omega_{V} \rightarrow V, \pi_{k}\left(v_{1}, v_{2}, \ldots\right)=v_{k}$, constitute a Markov process with respect to the natural filtration $\mathscr{F}_{k}$ (generated by the coordinates $\pi_{1}, \ldots, \pi_{k}$ ) with transition probabilities $P_{k}$ and initial distribution $\mu$. That is, such that for any $l \leq k$ and any measurable $f: V \rightarrow \mathbb{R}^{+}$,

$$
\mathbb{E}_{\mu}\left[f \circ \pi_{k} \mid \mathscr{F}_{l}\right](\omega)=\left(P^{k-l} f\right)\left(v_{l}\right)
$$

for $P_{\mu}$ almost all $\omega$, where $\left(P^{k} f\right)(v)=\int_{V} f(w) P_{k}(d w \mid v)$ is the semigroup of positive linear operators corresponding to the kernel $\left(P_{k}\right)_{k \in \mathbb{N}}$ and $P_{\mu}$ is given on cylinders $U=\left\{\omega \in \Omega_{V}: \pi_{i}(\omega) \in A_{i}, i=0,1, \ldots, k\right\}$ by

$$
P_{\mu}(U)=\int_{A_{0}} \int_{A_{1}} \cdots \int_{A_{k}} P\left(d v_{k} \mid v_{k-1}\right) \cdots P\left(d v_{2} \mid v_{1}\right) \mu\left(d v_{1}\right) .
$$

Notice that $\left(\pi_{0}\right)_{*} P_{\mu}=\mu$.

The measure $P_{\mu}$ is shift invariant if and only if $\mu$ is stationary, or $P^{k}$ invariant, for $k=1,2, \ldots$; that is, if

$$
\mu=\int_{V} P_{k}(\cdot \mid v) \mu(d v)
$$

A bounded measurable function $f$ on $V$ is said to be $P^{k}$-invariant if $P^{k} f=f$ $\mu$-almost everywhere, for all $k$. A stationary measure $\mu$ is ergodic if invariant functions are $\mu$-almost everywhere constant. It can be shown that $\mu$ is ergodic 
if and only if the shift map on $\Omega_{V}$ is ergodic for the invariant measure $P_{\mu}$. This is also equivalent to invariance in the sense

$$
\mu=\int_{X}\left(\Psi_{x}\right)_{*} \mu d \lambda(x)
$$

(See below. Also see [Ki] or [Ar].)

Suppose, now, that $\mu$ is a measure on $V$ such that $v:=\lambda \otimes \mu$ is a $T$-invariant measure on $E$. The next remark is that $\mu$ is an invariant measure of the random billiard, hence also a stationary measure for the associated Markov chain.

Proposition 2.1. Suppose that $\mu$ is a measure on $V$ such that $v=\lambda \otimes \mu$ is a $T$-invariant measure on $E$. Then $\mu$ is an invariant measure of the random billiard. In particular, $\mu$ is a stationary measure of the associated Markov chain.

Proof. Let $f$ be any $\mu$-integrable function on $V$. Then, from the equation $T_{*} v=v$ applied to $f \circ \pi_{2}$, we obtain

$$
\begin{aligned}
\mu(f) & =v\left(f \circ \pi_{2}\right)=T_{*} \nu\left(f \circ \pi_{2}\right)=v\left(f \circ \pi_{2} \circ T\right) \\
& =\int_{X} \int_{V} f\left(\pi_{2}(T(x, v))\right) d \mu(v) d \lambda(x) \\
& =\int_{X} \int_{V} f\left(\Psi_{x}(v)\right) d \mu(v) d \lambda(x)=\int_{0}^{1}\left(\Psi_{x}\right)_{*} \mu(f) d \lambda(x) .
\end{aligned}
$$

Since $f$ is arbitrary, we have $\mu=\int_{X}\left(\Psi_{x}\right)_{*} \mu d \lambda(x)$ as claimed. That $\mu$ is a stationary measure for the Markov chain is now a standard fact. We show it here for the sake of completeness. The claim is that

$$
\mu(A)=\int_{V} P(A \mid v) d \mu(v),
$$

for all measurable $A \subset V$. This is a consequence of the following calculation:

$$
\begin{aligned}
\int_{V} P(A \mid v) d \mu(v) & =\int_{V} \int_{X} \chi_{A}\left(\Psi_{x}(v)\right) d \lambda(x) d \mu(v) \\
& =\int_{X} \int_{V} \chi_{A}\left(\Psi_{x}(v)\right) d \mu(v) d \lambda(x)=\int_{X}\left(\Psi_{x}\right)_{*} \mu(A) d \lambda(x) \\
& =\mu(A),
\end{aligned}
$$

for all measurable $A \in V$.

From now on, we resume the earlier convention that $\mu$ denotes the velocity component of the Liouville measure on $E$. 


\section{Examples}

We illustrate the concepts introduced above with a few simple examples. The simplest, shown in Figure 2, was discussed in the introduction.

3.1. Three-dimensional boxes. The billiard cell is defined by the surface of a parallelepiped without its top face, with sides $h$ (height), $b_{1}$ and $b_{2}$. Using the vectors $e_{1}, e_{2}, n$ as in Figure 8, the base of the parallelepiped is oriented as in Figure 9.

If $v=v_{1}^{\prime} u_{1}+v_{2}^{\prime} u_{2}-v_{3} n$ is an incoming velocity expressed in the orthonormal frame aligned with the box, then the velocity after reflection is

$$
v=\varepsilon_{1} v_{1}^{\prime} u_{1}+\varepsilon_{2} v_{2}^{\prime} u_{2}+v_{3} n,
$$

where $\varepsilon_{1}$ and $\varepsilon_{2}$ are independent random variables taking values in $\{1,-1\}$ with probabilities to be specified. Write

$$
A=\left(\begin{array}{rr}
\cos \alpha & -\sin \alpha \\
\sin \alpha & \cos \alpha
\end{array}\right)
$$

and let $v=v_{1} e_{1}+v_{2} e_{2}-v_{3} n$ be an incoming velocity, now expressed in the frame of the channel. The velocity after reflection can now be written as a random variable $V\left(\varepsilon_{1}, \varepsilon_{2}\right)=w_{1} e_{1}+w_{2} e_{2}+v_{3} n$ such that

$$
\left(\begin{array}{l}
w_{1} \\
w_{2}
\end{array}\right)=A\left(\begin{array}{ll}
\varepsilon_{1} & 0 \\
0 & \varepsilon_{2}
\end{array}\right) A^{t}\left(\begin{array}{l}
v_{1} \\
v_{2}
\end{array}\right)
$$

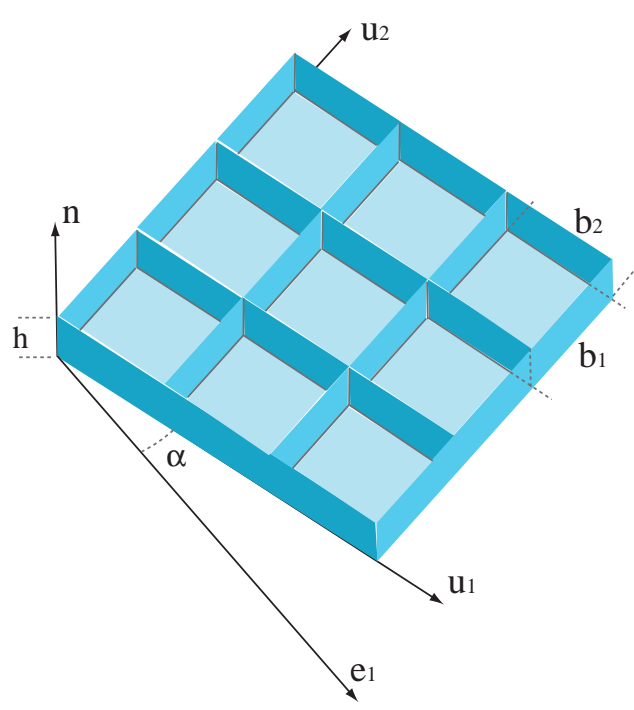

Figure 9. Geometry of microcubicles. 
This can also be written as follows. Let $u$ be the orthogonal projection of $v$ to the linear span of $\left\{e_{1}, e_{2}\right\}$. Then $v=\|u\|\left(\cos \beta e_{1}+\sin \beta e_{2}\right)-v_{3} n$ ( $\beta$ is defined by this expression) and $V\left(\varepsilon_{1}, \varepsilon_{2}\right)=\|u\| U\left(\varepsilon_{1}, \varepsilon_{2}\right)+v_{3} n$, where

$U\left(\varepsilon_{1}, \varepsilon_{2}\right)=\frac{\varepsilon_{1}+\varepsilon_{2}}{2}\left(\cos \beta e_{1}+\sin \beta e_{2}\right)+\frac{\varepsilon_{1}-\varepsilon_{2}}{2}\left(\cos (2 \alpha-\beta) e_{1}+\sin (2 \alpha-\beta) e_{2}\right)$.

For a given initial $v$, the velocity after any later collision of the random flight in a cylindrical channel is one of the four vectors $\pm u+v_{3} n, \pm u^{\prime}+v_{3} n$, where $u^{\prime}$ is defined by

$$
u^{\prime}=\|u\|\left(\cos (2 \alpha-\beta) e_{1}+\sin (2 \alpha-\beta) e_{2}\right)
$$

and the frame vectors are based at the collision point.

We assume that $v$ is not parallel to $u_{1}$ or $u_{2}$ (the easier case of $v$ parallel to either vector can be treated separately). Let

$$
\frac{2 h\langle v, n\rangle}{b_{i}\left\langle v, u_{i}\right\rangle}=: k_{i}(v)+s_{i}(v)
$$

where $k_{i}$ is the integer part and $s_{i}$ is the fractional part of the number on the left-hand side of the equation. Define $p_{i}(v)=s_{i}(v)$ if $k_{i}(v)$ is odd and $p_{i}(v)=$ $1-s_{i}(v)$ if $k_{i}(v)$ is even. The probability that $\varepsilon_{i}=1$, given that $v$ is the precollision velocity, is $p_{i}(v)$.

For simplicity of notation, we write below $p_{i}(u)$ instead of $p_{i}\left(u \pm v_{3} n\right)$. We make explicit the dependence of $\varepsilon_{i}$ on $w \in\left\{ \pm u+v_{3} n, \pm u^{\prime}+v_{3} n\right\}$ (the precollision velocity) by writing $\varepsilon_{i}(w)$. Define $k_{i}, s_{i}$ as the integral and fractional

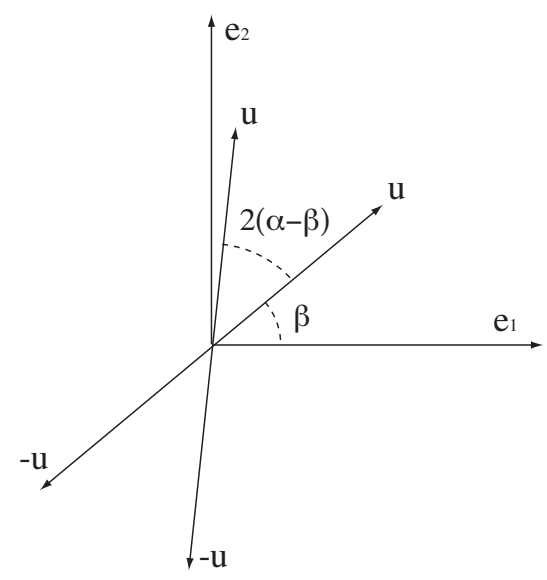

Figure 10. Possible values of the orthogonal projection of the reflected velocity. 


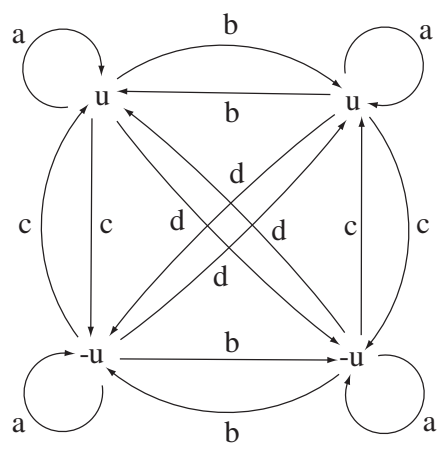

Figure 11. Transition probabilities for the velocity process.

parts of the numbers

$$
\frac{2 h v_{3} / \sqrt{1-v_{3}^{2}}}{b_{1} \cos (\beta-\alpha)}=k_{1}+s_{1}, \quad \frac{2 h v_{3} / \sqrt{1-v_{3}^{2}}}{b_{2} \sin (\beta-\alpha)}=k_{2}+s_{2} .
$$

It can now be calculated that $p_{i}(u)=p_{i}\left(u^{\prime}\right)=p_{i}(-u)=p_{i}\left(-u^{\prime}\right)=: p_{i}$, where

$$
p_{i}= \begin{cases}s_{i} & \text { if } k_{i} \text { is even, } \\ 1-s_{i} & \text { if } k_{i} \text { is odd. }\end{cases}
$$

Setting $a=p_{1} p_{2}, b=p_{1}\left(1-p_{2}\right), c=\left(1-p_{1}\right) p_{2}, d=\left(1-p_{1}\right)\left(1-p_{2}\right)$, the transition probabilities can now be seen to be as shown in Figure 11 .

The random walk on the real line, obtained by projecting the random flight inside the cylinder on a line parallel to $e_{2}$, can be described as follows. We suppose that the initial velocity is $v=u-v_{3} n$. From any particular time and position, a particle moves a distance $\delta$, which can be one of the four values: $\delta_{1}, \delta_{2}, \delta_{3}=-\delta_{2}, \delta_{4}=-\delta_{1}$, where

$$
\begin{aligned}
& \delta_{1}=\frac{2 r v_{3}\|u\| \sin \beta}{v_{3}^{2}+\|u\|^{2} \cos ^{2} \beta}, \\
& \delta_{2}=\frac{2 r v_{3}\|u\| \sin (2 \alpha-\beta)}{v_{3}^{2}+\|u\|^{2} \cos ^{2}(2 \alpha-\beta)} .
\end{aligned}
$$

A jump by $\pm \delta_{i}$ takes time $\tau_{i}$, where

$$
\begin{aligned}
\tau_{1} & =\frac{2 r v_{3}}{v_{3}^{2}+\|u\|^{2} \cos ^{2} \beta}, \\
\tau_{2} & =\frac{2 r v_{3}}{v_{3}^{2}+\|u\|^{2} \cos ^{2}(2 \alpha-\beta)} .
\end{aligned}
$$


Jumps of different lengths occur with probabilities specified by the transition matrix

$$
P=\left(p\left(\delta_{i} \mid \delta_{j}\right)\right)=\left(\begin{array}{cccc}
a & b & c & d \\
b & a & d & c \\
c & d & a & b \\
d & c & b & a
\end{array}\right)
$$

where $a, b, c, d$ are as above. The spectral decomposition of the transition matrix is easily obtained. If $R$ is the orthogonal matrix

$$
R=\frac{1}{2}\left(\begin{array}{rrrr}
1 & 1 & 1 & 1 \\
1 & 1 & -1 & -1 \\
1 & -1 & 1 & -1 \\
1 & -1 & -1 & 1
\end{array}\right)
$$

then $P=R D R^{t}$, where $D=\operatorname{diag}\left(\lambda_{1}, \lambda_{2}, \lambda_{3}, \lambda_{4}\right)$, with

$$
\begin{aligned}
& \lambda_{1}=a+b+c+d=1, \\
& \lambda_{2}=a+b-c-d=2 p_{1}-1, \\
& \lambda_{3}=a-b+c-d=2 p_{2}-1, \\
& \lambda_{3}=a-b-c+d=\left(2 p_{1}-1\right)\left(2 p_{2}-1\right) .
\end{aligned}
$$

In particular, the stationary distribution assigns equal probability to each of the $\delta_{i}$. By allowing different values of the initial velocity we can recover the geometric parameters, $b_{1} / h, b_{2} / h, \alpha$, from the spectrum.

3.2. Cavities and effusion. The example we give now is in a sense more representative than those seen so far in that the measure $\mu$ is ergodic, as explained later. Furthermore, its Markov operator admits a simple approximation that will allow for some explicit calculations in Section 7. The approximate operator, which by itself does not correspond to any random billiard system, is defined by

$$
P_{\text {Maxwell }}=(1-\alpha) P_{\mu}+\alpha I,
$$

where $\alpha$ is a constant in $(0,1)$ and $P_{\mu}(d w \mid v)=d \mu(w)$. This is a model of gas-solid interaction proposed by Maxwell well-known in the gas kinetics and Boltzmann equation literature (except for the fact that we do not include an exponential term involving temperature) [C1]. The interpretation is that a particle will reflect in mirror-like fashion with probability $\alpha$, and with probability $1-\alpha$ it forgets the pre-collision velocity and rebounds along a random direction specified by the probability $\mu$.

Notice that $P_{\mu}$ can be described as the orthogonal projection on the onedimensional subspace spanned by $\mu$ in the Hilbert space, $H$, of signed measures 


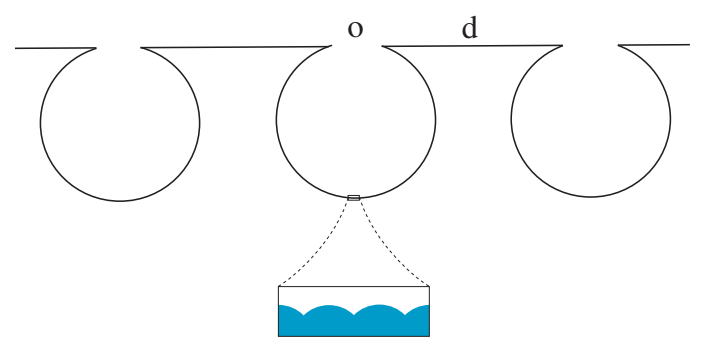

Figure 12. Randomizing cavity; $o$ is the length of the open side and $d$ is the length of the mirror-reflecting, flat side. The inside of the circular cavity is lined with some ergodic microgeometry.

with square integrable densities with respect to $\mu$. Then

$$
P_{\text {Maxwell }}=P_{\mu}+\alpha P_{\mu^{\perp}}
$$

where $\mu^{\perp}$ denotes the subspace of $H$ orthogonal to $\mu$.

We now show in what sense $P_{\text {Maxwell }}$ can be regarded as approximating the Markov operator of an actual billiard microgeometry. (Proposition 3.1 will not be used later in the paper other than for its heuristic value, so we only give a sketch of the proof.) Consider the contour shown in Figure 12. It represents an array of billiard cells consisting of circular cavities with a small opening on top. The inner surface of each cavity is itself lined with some other kind of microgeometry at a much smaller scale. The bumps lining the inner surface of the cavity are assumed to be so small that collisions with it are well approximated by a "second order" random billiard map, which we denote by $\Psi$. We assume that the associated Markov operator, $P_{\text {bumps }}$, is ergodic and aperiodic (see Section 5). In particular, $P_{\text {bumps }}^{k}$ converges to $\mu$ as $k \rightarrow \infty$. This is the case for the particular example of circular bumps shown in the figure, according to the ergodicity criterion given in Section 5 .

The random dynamical system, $\Phi$, is defined as follows. Let $v$ be an initial velocity and $x \in[0,1]$ a random point on the open side of the cavity chosen with probability $\lambda$. Set $a=o / l$, where $l$ is the perimeter of the circular cavity and $o$ is the length of its open side. With probability 1, a particle that falls into the cavity leaves it after a finite number, $\#_{a}$, of collisions. This number is a random variable depending on $v$. It can be shown that the limit, as $a \rightarrow 0$, of the probability of the event $\left\{\#_{a}<n\right\}$ is 0 for each finite $n$. Let $\Theta_{1}, \Theta_{2}, \ldots, \Theta_{\#_{a}}$ be the post-collision random angles inside the cavity, measured with respect to the tangent line at each (random) collision point. Let $W$ denote the random vector in $\mathbb{R}^{2}$ corresponding to the angle $\Theta_{\#_{a}}$. Then $\Phi(v)$ is the angle of $W$ measured relative to the direction parallel to open side. For small $a, \Phi(v)$ is very nearly $\Theta_{\# a}$. 
Notice that $\Theta_{i}$ is both the post-collision velocity of the $i$-th collision and the pre-collision velocity of the $i+1$-st collision. (This might not hold were the cavities not circular.) Therefore, $P_{\text {bumps }}^{k}$ actually describes the probability distribution of post-collision velocity after $k$ collisions inside the cavity.

Let, now, $P_{a}$ be the Markov operator of the random billiard conditioned on the particle actually entering the cavity. This is an event of probability $o /(o+d)$. Let $\alpha=d /(o+d)$ be the probability of an incident particle being reflected specularly. Then, the Markov operator associated to $\Phi$ can be written as $P=$ $(1-\alpha) P_{a}+\alpha I$, where $I$ is the identity operator. By the above argument, $P_{a}=P_{\text {bumps }}^{\#_{a}}$. Thus we conclude:

Proposition 3.1. Denote by $P_{a}(\cdot \mid v)=P_{\text {cav }}(\cdot \mid v)$ the conditional measures for a cavity with ratio o $l l=a$. Suppose that $\Psi$ has a unique stationary measure, $\mu$, and that the conditional measures are absolutely continuous with respect to $\mu$. (See Section 5; the "microbumps" of Figure 12 are one example for which these assumptions hold.) Then, for $\mu$-a.e. $v \in V, \lim _{a \rightarrow 0} P_{a}(\cdot \mid v)=\mu$.

Thus, it makes sense to think of $P_{\text {Maxwell }}=(1-\alpha) P_{\mu}+\alpha I$ as an approximation to the Markov operator of the random billiard of Figure 12, for a small opening at the top.

Another interpretation of Proposition 3.1 has to do with the phenomenon of effusion from cavities. If we imagine the circular cavity filled with gas at low pressure, then gas will escape through the small opening with a distribution of directions given by the probability density $d \mu / d \theta=\frac{1}{2} \sin \theta$. Here $\theta$ is the angle measured counterclockwise from the direction parallel to the open side. In particular, the probability of leaving at shallow angles approaches 0 .

\section{Reversibility and self-adjointness}

The deterministic billiard system has the property of being reversible. In dimension 2, this means:

$$
T(x, \theta)=(y, \phi) \Longleftrightarrow T(y, \pi-\phi)=(x, \pi-\theta),
$$

and in dimension 3 , replace $v$ for $\theta,-w$ for $\pi-\phi$, etc, in the above expression. This can also be written as $T^{-1} \circ J=J \circ T$, where $J(x, v)=(x,-v)$ is the flip map. If $\Phi_{t}$ is a flow describing the time evolution of, say, a conservative mechanical system on its phase space or a geodesic flow, then reversibility means that $\Phi_{t}(x, v)=(y, w) \Longleftrightarrow \Phi_{t}(y,-w)=(x,-v)$ for all $(x, v)$ and $t$. In this case, $J \circ \Phi_{t}=\Phi_{-t} \circ J$.

Reversibility of the deterministic billiard system implies reversibility of the random billiard as well, in the following sense. First recall that a Markov process 
with state space $V$ and transition probability measure $P$ is said to be reversible with respect to a probability measure $\mu$ on $X$ if

$$
P(d w \mid v) d \mu(v)=P(d v \mid w) d \mu(w),
$$

as measures on $V \times V$. More precisely,

$$
\int_{V} \int_{V} f(v, w) P(d w \mid v) d \mu(v)=\int_{V} \int_{V} f(v, w) P(d v \mid w) d \mu(w)
$$

for any integrable function $f(v, w)$ on $V^{2}$.

PROPOSITION 4.1. The Markov process of a random billiard is reversible.

Proof. We actually show that $P(d w \mid v) d \mu(v)=P(d v \mid w) d \mu(w)$ for any measure $\mu$ on $V$ such that $v:=\lambda \otimes \mu$ is invariant under the billiard first return map $T: E \rightarrow E$ and the flip map $J$. First observe that

$$
\begin{aligned}
\int_{V} \int_{V} f(v, w) P(d w \mid v) d \mu(v) & =\int_{V} \int_{X} f\left(v, \Psi_{x}(v)\right) d \lambda(x) d \mu(v) \\
& =\int_{E} F(\xi, T(\xi)) d v(\xi),
\end{aligned}
$$

where $F\left(\xi_{1}, \xi_{2}\right)=f\left(\pi_{2}\left(\xi_{1}\right), \pi_{2}\left(\xi_{2}\right)\right)$ for all $\xi_{1}, \xi_{2} \in E$. Therefore, the claim amounts to the property

$$
\int_{E} F(\xi, T(\xi)) d v(\xi)=\int_{E} F(T(\xi), \xi) d v(\xi) .
$$

But this is a consequence of the invariance of $v$ under $T^{-1}$ and the flip map.

Notice the following well-known consequence of reversibility of the Markov process with respect to the stationary measure $\mu$.

Proposition 4.2. Let $\langle f, g\rangle=\int_{0}^{\pi} f \bar{g} d \mu$ denote the inner product on the Hilbert space $H=L^{2}(V, \mu)$. Then, the linear operator $P$ on $H$ defined by

$$
(P f)(v):=\int_{X} f\left(\Psi_{x}(v)\right) d \lambda(x)
$$

is self-adjoint.

We regard $H$ as the space of densities of signed measure on $V$ absolutely continuous with respect to $\mu$. With this in mind, we write $P \eta$ for the action of the Markov operator $P$ on a probability measure $\eta$ on $V$. If $\eta$ describes the probability distribution of incoming directions of a particle, then $P \eta$ is interpreted as the probability distribution of reflected directions. 


\section{Ergodicity}

Given a Markov process with state space $V$ and transition probability measure $P$, let $P_{n}(A \mid v)$ denote the transition probability measure after $n$ steps. We are interested in the convergence of $P_{n}(A \mid v)$ to $\mu(A)$, where $\mu$ is the velocity component of the Liouville measure $v$ on $E$. Recall that $\mu$ is a stationary measure for $P$.

Lemma 5.1. Suppose that a measurable $A \subset V$ satisfies $P(A \mid v)=1$ for $\mu$-a.e. $v \in A$. Let $\bar{A}=X \times A$. Then, after possibly changing $A$ on a set of measure 0 , we have that $\bar{A}$ is $T$-invariant.

Proof. For almost all $v \in A, \lambda\left(\left\{x \in X: \Psi_{x}(v) \in A\right\}\right)=P(A \mid v)=1$. Consequently, for almost all $(x, v) \in \bar{A}$, we have $T(x, v) \in \bar{A}$. By changing $\bar{A}$ on a set of measure zero it can be insured that $\bar{A}$ is $T$-invariant.

We say that the process is indecomposable if any measurable set $A \subset V$ such that $P(A \mid v)=1$ for almost all $v \in A$ either has measure zero or its complement has measure zero.

Corollary 5.2. Suppose that $T: E \rightarrow E$ is ergodic with respect to the Liouville measure. Then the Markov process of the random billiard is indecomposable.

Proof. Apply the previous proposition to $\bar{A}=X \times A$, where $P(A \mid v)=1$ for $\mu$-a.e. $v$ to conclude that $\bar{A}=X \times A$ has measure either zero or one. Therefore, $A$ has measure either zero or one.

COROLlary 5.3. If $T: E \rightarrow E$ is ergodic (in particular, if the billiard $d y$ namical system on the closed table, with its top side included, is ergodic), then $\mu$ is the unique stationary distribution of the billiard Markov operator, and the Markov process with initial distribution $\mu$ is ergodic.

Proof. See [Br], Theorem 7.16.

Applying the previous result to powers of $T$, gives the next proposition.

Proposition 5.4. Suppose that all the iterates $T^{n}$ of $T$ are ergodic. Then Markov process is aperiodic, that is, $V$ is indecomposable for all the iterates $P^{n}(\cdot \mid v), n=1,2, \ldots$.

Corollary 5.5. Suppose that $T^{n}$ is ergodic for $n=1,2,3, \ldots$, and that $P(\cdot \mid v)$ is absolutely continuous with respect to $\mu$, for almost all $v$. Then

$$
\lim _{n \rightarrow \infty} P^{n}(A \mid v)=\mu(A)
$$

for all measurable $A$ and almost all $v \in V$. 
ProOF. This is a consequence of $[\mathrm{Br}]$, Theorem 7.18.

As an example, if the doubling of the billiard table at its open side gives a Sinai (dispersing) billiard, then the Markov process has a unique stationary measure given by $\mu$.

It is to be expected that ergodicity and aperiodicity should hold under very general conditions. It is certainly clear that it is a much more general (and easier to prove) property for the random billiard than it is for the corresponding deterministic billiard. We give next a sufficient condition that is easy to verify in many examples.

We say that $p$ is a point of maximal height of the (open) billiard cell if the entire cell is contained in one of the two closed half-spaces bounded by the plane through $p$ parallel to the distinguished, or top, side. We also say that the billiard is nondegenerate at $p$ if the height function (relative to the normal direction to the open side) is nondegenerate there. This means that at the point $p$ of maximal height the billiard surface, or curve, the curvature is defined and nonzero.

Proposition 5.6. Suppose that the billiard table contains a point of maximal height at which the billiard is nondegenerate. Then the associated random billiard is ergodic and aperiodic, and it admits a unique stationary probability measure, which is $\mu$ (the velocity factor of the Liouville measure).

Proof. Given $A \subset V$ measurable, define $\mathscr{I}(A)=\left\{\Psi_{x}(v): x \in X, v \in A\right\} \subset V$. If the billiard table is differentiable at a point $p$ of maximal height, then $A \subset \mathscr{I}(A)$, since $v=\Psi_{x}(v)$ for an $x$ such that the ray with initial condition $(x, v)$ reflects at $p$. We claim that, for all $v \in V$,

$$
V=\bigcup_{n=1}^{\infty} g^{n}(v) .
$$

Granted the claim, it follows that $P^{n}$ is indecomposable for $n=1,2, \ldots$ In fact, let $A \subset V$ satisfy $P(A \mid v)=1$ for $\mu$-a.e. $v \in A$. By Lemma 5.1, $\bar{A}=X \times A$ is $T$-invariant. Consequently, for almost all $v \in A, \Phi^{n}(v) \subset A$, up to sets of measure 0 . Therefore, $A=V$ up to a set of measure 0 .

We now turn to the proof of the claim. The description below applies to dimension 3, although it should be clear what modifications are need for dimension 2. The surface boundary of the billiard table will be denoted by $M$. This is a smooth surface near $p$. Recall that $V$ is the open hemisphere of unit radius. Let $\Pi: V \rightarrow D$ denote the orthogonal projection onto the open unit disc. The essential point is that, for any compact subset, $K \subset V$, there is $\varepsilon>0$ such that for any $v \in K$, the set $\Pi(\mathscr{F}(v))$ contains a disc of radius $\varepsilon$ centered at $\Pi(v)$. To see this, it suffices to show that the Jacobian of $x \in \mathbb{T}^{2} \mapsto \Pi\left(\Psi_{x}(v)\right)$ is uniformly bounded away from 0 for $v$ in any compact subset of $V$. 
Let $\gamma(s)$ be a differentiable curve in $\mathbb{T}^{2}$ such that the ray with initial condition $(\gamma(0), v)$ hits $M$ at $p$. By varying $s$, for $s$ small enough, a differentiable path, $\eta$, through $p$ is traced on $M$. The reflected angle is given by

$$
\Psi_{\gamma(s)}(v)=v-2\langle v, n(\eta(s))\rangle n(\eta(s)) .
$$

Let $G: M \rightarrow S^{2}$ denote the Gauss map of $M$ near $p$. Note that, by definition, the differential of $G$ at $p$ is

$$
d G_{p} \eta^{\prime}(0)=\left.\frac{d(n \circ \eta)}{d s}\right|_{s=0}
$$

We can now write

$$
\begin{aligned}
\left.\frac{d}{d s} \Pi \circ \Psi_{\gamma(s)}(v)\right|_{s=0} & =\Pi\left(-2\left\langle v, d G_{p} \eta^{\prime}(0)\right\rangle n(p)-2\langle v, n(p)\rangle d G_{p} \eta^{\prime}(0)\right) \\
& =-2\langle v, n(p)\rangle \Pi d G_{p} \eta^{\prime}(0) .
\end{aligned}
$$

The norm of $\Pi$ at $n$ is 1 and $\gamma^{\prime}(0)=\eta^{\prime}(0)$. Consequently,

$$
\left\|\left.\frac{d}{d s} \Pi \circ \Psi_{\gamma(s)}(v)\right|_{s=0}\right\| \geq 2|\langle v, n(p)\rangle| \min \left\{k_{1}, k_{2}\right\}\left\|\gamma^{\prime}(0)\right\|,
$$

where $k_{1}$ and $k_{2}$ are the principal curvatures of $M$ at $p$, that is, the eigenvalues of $d G_{p}$, which are necessarily positive. Since the term $|\langle v, n(p)\rangle|$ is bounded away from 0 for all $v$ on a compact $K \subset V$, the claim holds.

We have so far assumed that the particle undergoing a random flight is pointlike. There is obviously no loss of generality in making this assumption since a spherical billiard ball can be regarded as point-like by following the motion of its center. If we wish to vary the size of the probing particle, then the problem we are studying is the same as having a one parameter family of microgeometries parametrized by the particle radius, and billiard balls that are point-like. Figure 13 illustrates this. The thick contour represents the actual surface relief, and the thin line represents this geometry as viewed by the center of a disc-like billiard ball.

It is interesting to remark that if $p$ is a point of maximal height at which the billiard is not-differentiable, say, a cone point, then viewed by the center of a spherical particle of positive radius, there is a point of maximal height (just above $p$ ) which is nondegenerate. Thus, for example, the comb geometry of Figure 13 does not correspond to an ergodic random billiard, but when probed

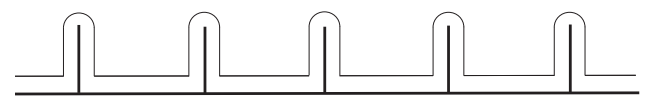

Figure 13. Microgeometry for positive particle radius. 
by particles of arbitrary nonzero radius, the thickened table (represented by the thin line in the figure) does.

\section{Spectrum}

A fundamental issue to address is the relationship between the geometry of the billiard cell and the spectrum of the (self-adjoint) Markov operator $P$ on $L^{2}(V, \mu)$. We take here a first step in this direction.

As before, $\mu$ refers to the (velocity part of the) Liouville measure on $V$. Let $\pi_{2}: E \rightarrow V$ denote the projection map. By duality, $P$ acts on signed measures on $V$ with square integrable densities with respect to $\mu$. If $\eta$ is such a measure, we write $\eta P$. (The notation $P \eta$ was also used earlier.)

\subsection{Generalities.}

LEMMA 6.1. Let $\varphi, \psi \in L^{2}(V, \mu)$, and $\eta$ the signed measure such that $d \eta=$ $\varphi d \mu$. Then

$$
\int_{V} \psi d(\eta P)=\int_{E}\left(\psi \circ \pi_{2}\right)\left(\varphi \circ \pi_{2} \circ T^{-1}\right) d \nu
$$

Proof. Let $\psi$ be any element of $L^{2}(V, \mu)$. Then

$$
\begin{aligned}
\int_{V} \psi \frac{d(\eta P)}{d \mu} d \mu & =\int_{V} \psi d\left[\left(\pi_{2} \circ T\right)_{*} \lambda \otimes \eta\right]=\int_{E} \psi \circ \pi_{2} \circ T d(\lambda \otimes \eta) \\
& =\int_{E}\left(\psi \circ \pi_{2} \circ T\right)\left(\varphi \circ \pi_{2}\right) d \nu \\
& =\int_{E}\left(\psi \circ \pi_{2}\right)\left(\varphi \circ \pi_{2} \circ T^{-1}\right) d \nu
\end{aligned}
$$

PROPOSITION 6.2. The spectrum of the Markov operator $P$ is contained in the interval $[-1,1]$, and 1 is an eigenvalue. If the associated Markov chain is ergodic, then 1 is a simple eigenvalue of $P$.

PROOF. Clearly, 1 is the eigenvalue associated to the stationary measure $\mu$, and is a simple eigenvalue if the Markov chain is ergodic. That $\|P\|_{2} \leq 1$ is shown by the following standard calculation:

$$
\|\eta P\|_{2}=\sup _{\psi \in L^{2}(V, \mu),\|\psi\|_{2}=1} \int_{V} \psi \frac{d(\eta P)}{d \mu} d \mu
$$




$$
\begin{aligned}
& =\sup _{\substack{\psi \in L^{2}(V, \mu) \\
\|\psi\|_{2}=1}} \int_{E}\left(\psi \circ \pi_{2}\right)\left(\varphi \circ \pi_{2} \circ T^{-1}\right) d v \\
& \leq \sup _{\substack{\psi \in L^{2}(V, \mu) \\
\|\psi\|_{2}=1}} \sqrt{\int_{E}\left(\psi \circ \pi_{2}\right)^{2} d v} \sqrt{\int_{E}\left(\varphi \circ \pi_{2} \circ T^{-1}\right)^{2} d v} \\
& =\sqrt{\int_{E}\left(\varphi \circ \pi_{2}\right)^{2} d v}=\sqrt{\int_{V} \varphi^{2} d \mu}=\|\eta\|_{2} .
\end{aligned}
$$

Therefore, $\|\eta P\|_{2} \leq\|\eta\|_{2}$, for all $\eta$. As $P$ is self-adjoint, its spectrum is real, contained in $[-1,1]$.

Proposition 6.3. Suppose that the conditional measure $P(\cdot \mid v)$ is absolutely continuous with respect to $\mu$, for $\mu$-almost every $v \in V$, and that the function $\kappa(w \mid v):=P(d w \mid v) / d \mu(v)$ (the Radon-Nikodým derivative of $P(\cdot \mid v)$ with respect to $\mu)$ is in $L^{2}\left(V^{2}, \mu \otimes \mu\right)$. Then, $P: H \rightarrow H$ is a Hilbert-Schmidt operator.

PROOF. Under these assumptions,

$$
(P f)(v)=\int_{V} f(w) \kappa(w \mid v) d \mu(w) .
$$

Since $\kappa$ is in $L^{2}\left(V^{2}, \mu \otimes \mu\right), P$ is a Hilbert-Schmidt operator.

6.2. Integral kernel and spectrum. We make at this point more specific and stronger assumptions about the deterministic billiard than we have so far. We restrict ourselves to the 2-dimensional case. One simple example for which all the assumptions to be made here hold is the billiard cell shown in Figure 14. It consists of two arcs of circle of positive curvature meeting at an angle $\gamma>\pi / 2$, and forming angles $\alpha$ and $\beta$ with the line segment representing the open side, where $\alpha, \beta \in[0, \pi / 2)$. Notice that every trajectory collides with the curved sides at most twice before returning to the open side. Also notice that for each initial condition $\left(x_{0}, \theta\right)$ at which the return map is differentiable, the function of $x$ defined by $F_{\theta}(x):=\Psi_{x}(\theta)$ is differentiable at $x_{0}$ and $\left|F_{\theta}^{\prime}\left(x_{0}\right)\right|$ is bounded away from 0 by a constant that depends on $\theta$ and on the curvatures of the two sides.

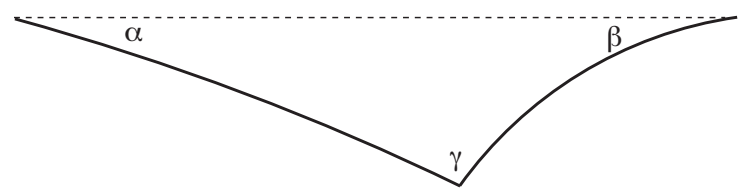

Figure 14. A simple example satisfying the requirements of this section. 
The results to be proved later under the following assumptions are by no means optimal. They should be expected to hold for much more general billiard geometries.

AsSumption 1. For each $\theta \in(0, \pi)$ there exists a countable set, $X_{\theta}=\left\{x_{i}^{\theta}\right\}$, where $0=x_{0}^{\theta}<x_{1}^{\theta}<\cdots<x_{l}^{\theta}=1$ and $l=l_{\theta} \leq \infty$, such that

(1) $F_{\theta}$ is differentiable and $F_{\theta}^{\prime} \neq 0$ over each interval $\left(x_{i}^{\theta}, x_{i+1}^{\theta}\right)$; and

(2) if $\mathscr{E}_{\theta}=\left\{\psi_{0}^{+}, \psi_{1}^{-}, \psi_{1}^{+}, \psi_{2}^{-}, \psi_{2}^{+}, \ldots\right\}$ denotes the set of right and left limits of $F_{\theta}$ at the $x_{i}^{\theta}$, then

$$
\sum_{x \in F_{\theta}^{-1}(\psi)}\left|F_{\theta}^{\prime}(x)\right|^{-1}<\infty
$$

for each $\psi \in(0, \pi) \backslash \mathscr{E}_{\theta}$ for which $F_{\theta}^{-1}(\psi)$ is nonempty.

ASSUMPTION 2. There exists a constant $c>0$ such that all sides have curvature bounded below by $c$. In particular, all sides are concave.

ASSUMPTION 3. Above a certain height, which is strictly below the height of the open side, the billiard table contour consists of two smooth, concave curves with nonzero curvature at the endpoints $p$ and $q$. (See Figure 15.) The angles $\alpha$ and $\beta$ lie in the interval $[0, \pi / 2)$.

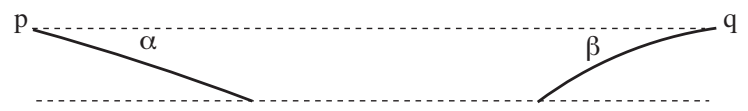

Figure 15. Assumption 3.

These assumptions are satisfied by the example of Figure 14. The number of $x_{i}^{\theta}$, not counting 0 and 1 , is at most 2 for each $\theta$, and $\left|F_{\theta}^{\prime}(x)\right|$ is bounded away from zero by a constant that depends on theta and on the curvatures of the arcs of circles. It should be noted that the sum increases with $1 / \sin \theta$ as $\theta$ approaches 0 and $\pi$.

Lemma 6.4. Fix $\theta \in(0, \pi)$. Under Assumption 1, the push-forward measure $\left(F_{\theta}\right)_{*} \lambda$ is absolutely continuous with respect to $\mu$. The Radon-Nikodým derivative, $\kappa(\psi \mid \theta)=\left[d\left(F_{\theta}\right)_{*} \lambda / d \mu\right](\psi)$, is given by

$$
\kappa(\psi \mid \theta)=\left\{\begin{array}{cl}
\frac{2}{\sin \psi} \sum_{x \in F_{\theta}^{-1}(\psi)}\left|F_{\theta}^{\prime}(x)\right|^{-1} & \text { if } F_{\theta}^{-1}(\psi) \text { is nonempty } \\
0 & \text { if } F_{\theta}^{-1}(\psi) \text { is empty }
\end{array}\right.
$$




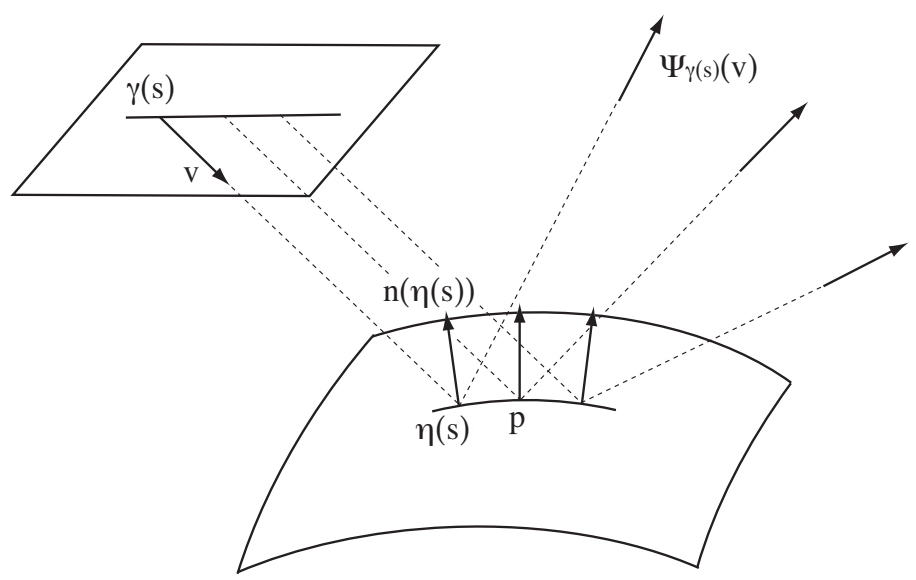

Figure 16. Reflection near a point of maximum height.

for all $\psi \in(0, \pi) \backslash \mathscr{E}_{\theta}$. If, moreover, $f$ is a bounded measurable function on $V$,

$$
\int_{0}^{1} f\left(F_{\theta}(x)\right) d \lambda(x)=\int_{0}^{\pi} f(\psi) \kappa(\psi \mid \theta) d \mu(\psi) .
$$

PROOF. This is an immediate consequence of the definitions.

Lemma 6.5. Under Assumption 2, for all $\theta \in(0, \pi)$ and $\psi \in(0, \pi) \backslash \mathscr{E}_{\theta}$, the function $\kappa(\psi \mid \theta)$ satisfies

$$
\kappa(\psi \mid \theta) \leq \frac{N / c}{\sin \psi \sin \theta} .
$$

Proof. Denote by $c(\theta)$ the infimum of $\left\{\left|F_{\theta}^{\prime}(x)\right|: x \in(0,1)\right\}$. From the expression of $\kappa$ given in Lemma 6.4, it follows that $\kappa(\psi \mid \theta) \leq 2 N /(c(\theta) \sin \psi)$. The lemma will be proved if we show that $c(\theta) \geq 2 c \sin \theta$.

Since all sides of the billiard table are strictly concave, we can estimate $\left|F_{\theta}^{\prime}(x)\right|$ by estimating the change of direction of the billiard trajectory due to the first collision only; the subsequent collisions can only magnify the angle variation to first order. Let $F(x)=F_{\theta, 1}(x)$ denote the angle immediately after the first collision, measured with respect to some direction, say, a tangent vector to the side at the point of first collision. (This choice is immaterial since we are interested in the derivative of $F(x)$.)

The situation can be pictured by imagining the two-dimensional version of Figure 16. In the present case, $p$ denotes the point of first collision and $\Psi_{\gamma(s)}(v)$ should be replaced with $F(\gamma(s))$. Also, the normal vector at $p$ is not necessarily normal to the open side, as is the case in that figure. We continue to denote by $\gamma(s)$ a differentiable curve such that $p_{0}=\gamma(0)$ is a point on the open side where $F$ is differentiable, and by $\eta(s)$ the curve traced on the side of first collision by 
the first segment of the billiard trajectory. If $v$ is, as in the figure, the unit vector representing the initial direction of the billiard trajectory, $\eta$ can be written as $\eta(s)=\gamma(s)+t(s) v$, for a positive differentiable function $t(s)$. The trajectory's direction immediately after the first collision now reads

$$
w(s)=v-2\langle v, n(\eta(s))\rangle n(\eta(s)) .
$$

Notice that $\left|F^{\prime}(x)\right|=\left\|w^{\prime}(0)\right\|$. Taking the square norm of $w^{\prime}(0)$, and using that $d n / d s=k(p) \eta^{\prime}(0)$, where $k(p)$ denotes the curvature of the billiard contour at $p$, gives

$$
\left\|w^{\prime}(0)\right\|^{2}=4 k(p)^{2}\left[\left\langle v, \frac{\eta^{\prime}(0)}{\left\|\eta^{\prime}(0)\right\|}\right\rangle^{2}+\langle v, n(p)\rangle^{2}\right]\left\|\eta^{\prime}(0)\right\|^{2} .
$$

The quantity between square brackets is the norm of $v$, since $\eta^{\prime}(0)$ and $n(p)$ are orthogonal, and $\left\|\eta^{\prime}(0)\right\|$ assumes its minimum value when $n(p)$ and $v$ are parallel, in which case $\left\|\eta^{\prime}(0)\right\|=\left\|\gamma^{\prime}(0)\right\| \sin \theta$. Therefore, $\left|F^{\prime}(x)\right|=\left\|w^{\prime}(0)\right\| \geq$ $2|k(p)| \sin \theta$, as needed to finish to proof.

We note the following simple geometric fact derived in the proof of Lemma 6.5.

LEMMA 6.6. Using the notations defined in the proof of Lemma 6.5, it holds that $\left\|w^{\prime}(0)\right\|=2|k(p)|\left\|\eta^{\prime}(0)\right\|$.

LEMMA 6.7. Under Assumptions 1, 2 and 3, $\kappa$ belongs to $L^{2}\left(V \times V, \mu^{2}\right)$.

PROOF. Due to Lemma 6.5, we only need to study the behavior of $\kappa$ for incident and reflected rays having angles close to 0 or $\pi$. It is sufficient to carry the analysis for angles close to 0 ; angles close to $\pi$ are similarly treated. There are four cases to study, depending on whether $\alpha$ and $\beta$ are positive or 0 . We consider only two: (i) $\alpha>0$ and $\beta>0$, and (ii) $\alpha=\beta=0$. The two remaining cases follow by applying the same arguments used for these.

Case (i). The exit angle $\psi$ can only be close to 0 when the incidence angle $\theta$ is close to $2 \alpha$ and $x$ is close to the left end point of the open side. We refer to this point as the left corner, and its opposite as the right corner. Therefore, we only need to study $\kappa(\psi \mid \theta)$ for $\theta$ near 0 and $2 \alpha$.

If $\theta_{0}$ is sufficiently small, then for all $0<\theta \leq \theta_{0}$ the function $F_{\theta}(x)$ has the following qualitative properties: it is smooth (assuming that the geometry of the billiard table is smooth near the ends), and it decreases monotonically from a maximum value, $\psi_{\max }^{\theta}$, to be estimated in a moment, to a minimum value, $\psi_{\min }^{\theta}=\theta+2 \beta$. Therefore, using Lemma 6.5 again, we arrive at the upper bound for $\kappa$ :

$$
\kappa(\psi \mid \theta) \leq \frac{1}{c \sin \psi \sin \theta} \chi_{\left[\psi_{\min }, \psi_{\max }\right]}(\psi)
$$


for $\theta \in\left(0, \theta_{0}\right)$ and a sufficiently small $\theta_{0}$. Here $\chi_{\left[\psi_{\min }, \psi_{\max }\right]}$ denotes the characteristic function of the interval $\left[\psi_{\min }, \psi_{\max }\right]$.

We claim that $\psi_{\max }-\psi_{\min } \leq C \sin \theta$, for some constant $C$. Suppose for the moment that this is the case. It easily follows that

$$
\int_{0}^{\theta_{0}} \int_{0}^{\pi} \kappa(\psi \mid \theta)^{2} d \mu(\psi) d \mu(\theta) \leq \frac{C c^{2} \theta_{0}}{2 \sin (2 \beta)} .
$$

Therefore, the proposition will be established once we justify the claim.

Using the expression of Lemma 6.6 gives

$$
\left|F_{\theta}^{\prime}(x)\right| \leq 2 K\left\|\eta^{\prime}(x)\right\|,
$$

where $K$ is the maximum value of the curvature of the side of the table contour making an angle $\beta$ with the open side and $\eta(x)$ is the curve traced along that side by the ray with initial condition $(x, \theta), x \in[0,1]$. Let $L=L(\theta)$ denote the length of this curve. Observe that

$$
\psi_{\max }-\psi_{\min }=\int_{0}^{1}\left|F_{\theta}^{\prime}(x)\right| d x .
$$

By elementary geometry, we have

$$
L=\int_{0}^{1}\left\|\eta^{\prime}(x)\right\| d x \leq 2 \sin \theta / \sin \beta .
$$

Therefore, $\psi_{\max }-\psi_{\min } \leq C \sin \theta$, where $C=4 K / \sin \beta$, proving the claim.

Still in case (i), it remains to consider $\theta$ near $2 \alpha$. When $\theta$ is close to $2 \alpha$ from below, and $x$ is near the left corner, $\psi$ is close to $\pi-2 \beta>0$. Therefore, only the interval $(2 \alpha, 2 \alpha+a)$, for an arbitrarily small $a>0$, needs to be considered. We take $a$ small enough that the exit angle $\psi$, after reflection on the concave side near the left corner, satisfies $0 \leq \psi \leq \pi / 4$ (in particular, $\psi \leq \sqrt{2} \sin \psi$ ), and also small enough that $(\sin \theta)^{-1}<S$, for a constant $S<\infty$, for all $\theta \in(2 \alpha, 2 \alpha+a)$.

The same argument used in the first part of this proof gives

$$
\kappa(\psi \mid \theta) \leq \frac{S}{c \sin \psi} \chi_{\left[\psi_{\min }, \psi_{\max }\right]}(\psi) \leq \frac{\sqrt{2} S}{c \psi} \chi_{\left[\psi_{\min }, \psi_{\max }\right]}(\psi),
$$

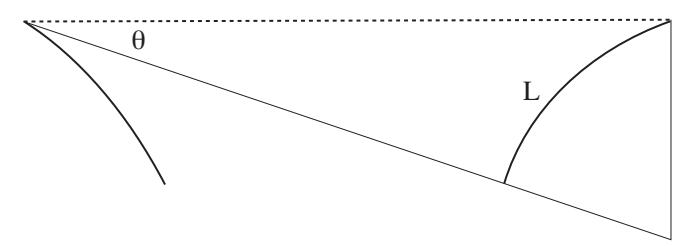

Figure 17. Definition of $L$. 
where it is understood that the maximum and minimum values of $\psi$ are functions of $\theta$. Therefore,

$$
\int_{2 \alpha}^{2 \alpha+a} \int_{0}^{\pi} \kappa(\psi \mid \theta)^{2} d \mu(\psi) d \mu(\theta) \leq \frac{2 S^{2}}{c^{2}} \int_{2 \alpha}^{2 \alpha+a} \ln \frac{\psi_{\max }}{\psi_{\min }} d \theta .
$$

The proof of case (i) will be concluded once we establish the following claim: if $\bar{k}$ denotes the maximum curvature of the side of the table the particle is reflecting from, then

$$
\frac{\psi_{\max }}{\psi_{\min }} \leq 1+\frac{8 \bar{k}}{\sin \alpha}
$$

for all $\theta \in(2 \alpha, 2 \alpha+a)$. We refer to Figure 18 .

In the figure, $L$ is the length of the segment of curve in the side of first reflection going from the left corner to the point of collision. Notice that the angle $\alpha(L)$ between the tangent at that point and the horizontal direction (parallel to the open side) satisfies $\alpha(L) \leq \alpha+2 \bar{k} L$. It also holds that

$$
L \leq 2 \sin \psi_{\min } / \sin \alpha \leq \frac{2}{\sin \alpha} \psi_{\min } .
$$

The maximum value of $\psi$ (the angle of reflection when the collision takes place at the left corner) is $\psi_{\max }=\theta-2 \alpha$. The minimum angle is $\psi_{\max }=\theta-2 \alpha(L)$. Therefore

$$
\psi_{\max }=\psi_{\min }+2(\alpha(L)-\alpha) \leq \psi_{\min }+\frac{8 \bar{k}}{\sin \alpha} \psi_{\min }
$$

and the claim about the ratio of these two angles holds.

Case (ii). We suppose now that $\alpha=\beta=0$. In this case, the exit angle $\psi$ can only approach 0 as $\theta$ itself approaches 0 . With Lemma 6.5 in mind, to prove that $\kappa$ is square integrable, it suffices to study what happens when $\theta$ is close to 0 . (The same argument treats the case of $\theta$ close to $\pi$.)

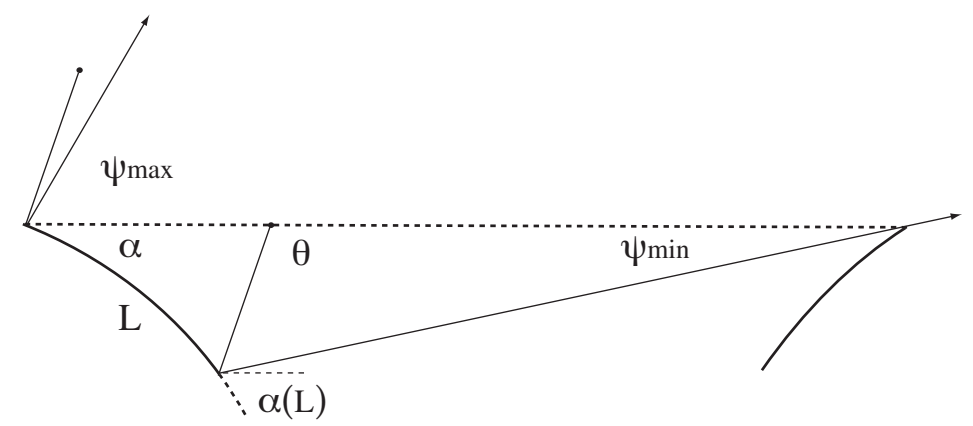

Figure 18. Exit at small angles. 
In case (i) the main part of the analysis concerned values of $\theta$ for which only one collision between particle and table boundary took place. This is no longer the case here. For a sufficiently small $\theta_{0}$, and $\theta \in\left(0, \theta_{0}\right)$, the function $\psi=F_{\theta}(x)$ has the qualitative properties shown in Figure 19. This is a piecewise smooth, continuous function, with two points of discontinuity of $F_{\theta}^{\prime}(x)$, denoted $x_{1}(\theta), x_{2}(\theta)$. For simplicity, we omit reference to $\theta$ in $x_{1}, x_{2}$. Over the intervals $\left(0, x_{1}\right)$ and $\left(x_{2}, 1\right)$ the function is monotone decreasing, and it is monotone increasing over $\left(x_{1}, x_{2}\right)$.

The discontinuities observed in this graph are due to the different types of trajectories, as follows. For a small $\theta$, if $x \in\left(x_{2}, 1\right)$, the particle will collide with the concave segment of wall adjacent to the right corner and exit the cave with an angle $\psi$ determined by this unique collision. At $x=x_{2}$, the trajectory first grazes the concave segment of wall adjacent to the left corner before hitting the concave right end wall and exiting. For $x \in\left(x_{1}, x_{2}\right)$, the trajectory first bounces off this left wall, then bounces off again at the right wall, then exits. For $x \in\left(0, x_{1}\right)$, there is only one collision, in this case with the left wall, before exiting.

We claim that the following estimates hold, for positive constants $A, B$ and $C$, and all $\theta \in\left(0, \theta_{0}\right)$ for a sufficiently small $\theta_{0}$ :

(1) $\psi_{\max } \leq A \theta^{1 / 2}$;

(2) $A^{-1} \theta^{2} \leq \psi_{\min }$;

(3) $\left|F_{\theta}^{\prime}(x)\right|^{-1} \leq B \theta^{-1 / 2}$, for all $x \in\left(x_{2}, 1\right)$;

(4) $\left|F_{\theta}^{\prime}(x)\right|^{-1} \leq C$, for all $x \in\left(0, x_{1}\right) \cup\left(x_{1}, x_{2}\right)$.

These estimates will be proven after it is shown how they give the desired result. An upper bound for $\kappa$ can now be given by

$$
\kappa(\psi \mid \theta) \leq \frac{1}{c \sin \psi}\left(2 C+B \theta^{-1 / 2}\right) \chi_{\left[\psi_{\min }, \psi_{\max }\right]}(\psi) .
$$

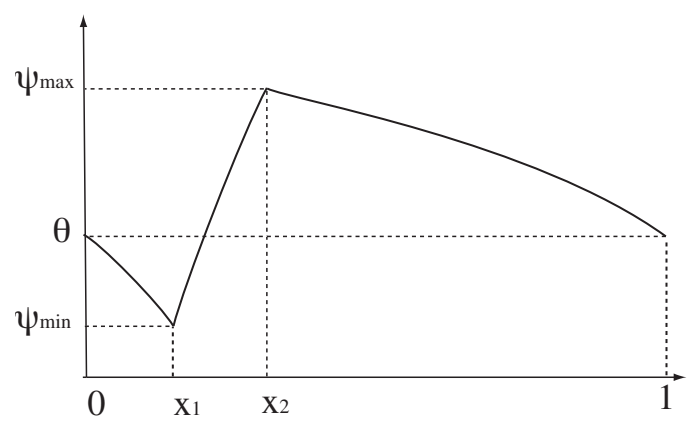

Figure 19. Qualitative features of $\psi=F_{\theta}(x)$ for small values of $\theta$. 
For small enough $\theta_{0}$ we have $2 C+B \theta^{-1 / 2} \leq 2 B \theta^{-1 / 2}$ and $\sin \psi \geq \sqrt{2} \psi$. This yields

$$
\begin{aligned}
\int_{0}^{\theta_{0}} \int_{0}^{\pi} \kappa(\psi \mid \theta)^{2} d \mu(\psi) d \mu(\theta) & \leq \frac{4 \sqrt{2} B^{2}}{c^{2}} \int_{0}^{\theta_{0}} \int_{0}^{\pi} \frac{1}{\psi} \chi_{\left[\psi_{\min }, \psi_{\max }\right]}(\psi) d \psi d \theta \\
& =\frac{4 \sqrt{2} B^{2}}{c^{2}} \int_{0}^{\theta_{0}} \ln \frac{\psi_{\max }}{\psi_{\min }} d \theta \\
& \leq \frac{4 \sqrt{2} B^{2}}{c^{2}} \int_{0}^{\theta_{0}} \ln \left(A^{2} \theta^{-3 / 2}\right) d \theta \\
& =\frac{6 \sqrt{2} B^{2} \theta_{0}}{c^{2}}\left(1-\ln \left(A^{-4 / 3} \theta_{0}\right)\right)<\infty
\end{aligned}
$$

It remains to verify the estimates enumerated above. Let $\xi(x, \theta)$ denote the angle between the normal to the boundary surface of the billiard and the vertical direction (i.e., the direction perpendicular to the open side, oriented toward the outside of the billiard cell). Then, for small values of $\theta$ and for $x \in\left(x_{2}, 1\right)$, we have $F_{\theta}(x)=\theta+2|\xi(x, \theta)|$. Ignoring the left wall and using concavity of the right wall, we obtain that $\psi_{\max } \leq \theta+2|\xi(0, \theta)|$. By an elementary geometric argument, it can be shown that $\lim _{\theta \rightarrow 0} \xi(0, \theta) / \sqrt{\theta}=\sqrt{2 k}$, where $k$ is the absolute value of the curvature of the right wall at the point of tangency with the open side. Therefore, we can find $D>0$ independent of $\theta$ such $D^{-1} \theta^{1 / 2} \leq$ $\xi(0, \theta) \leq D \theta^{1 / 2}$. (We note that if the right wall is replaced with a circumference of curvature $k$, then it can be shown by elementary geometry that $1-\cos \xi+$ $\sin \xi \tan \theta=k \sin \theta$, where $\xi=\xi(0, \theta)$. It follows from this that $\theta=\xi^{2} / 2 k+$ $o\left(\xi^{2}\right)$.) Therefore, there exists $A>0$ independent of $\theta$ such that $\psi_{\max } \leq A \theta^{1 / 2}$ as claimed.

The inequality $A^{-1} \theta^{2} \leq \psi_{\min }$ is proved in a similar way and we omit the details. Notice that the trajectory with exit angle $\psi_{\min }$ is the one that first reflects off the left wall at a point very close to the left corner and then grazes the right wall before exiting through the open side. In proving the second inequality, the roles of $\psi$ and $\theta$ are essentially reversed, giving $\theta^{2}$ instead of the $\theta^{1 / 2}$ of the first inequality.

The third inequality is obtained as follows. Fix $\theta$ and let $L(x), x \in\left(x_{2}, 1\right)$, denote the length of the segment of wall starting at the point $p(x, \theta)$ at which the trajectory with initial condition $(x, \theta)$ bounces off and ending at the right corner. Then it can be shown by elementary geometry that

$$
\left|L^{\prime}(x)\right|=\frac{\sin \theta}{\sin (\theta+\xi(x, \theta))} .
$$


We know from Lemma 6.6 that $\left|F_{\theta}^{\prime}(x)\right|=2|k(p(x, \theta))|\left|L^{\prime}(x)\right|$. Therefore, we can find a $D^{\prime}>0$ such that

$$
\begin{aligned}
\left|F_{\theta}^{\prime}(x)\right|^{-1} & =\frac{1}{2|k(p(x, \theta))|} \frac{\sin (\theta+\xi(x, \theta))}{\sin \theta} \leq D^{\prime}\left(1+\frac{\xi(x, \theta)}{\theta}\right) \\
& \leq D^{\prime}\left(1+\frac{\xi(0, \theta)}{\theta}\right) \leq D^{\prime}\left(1+\frac{D \theta^{1 / 2}}{\theta}\right) \leq B \theta^{-1 / 2},
\end{aligned}
$$

for some $B>0$, as claimed.

For $x \in\left(0, x_{2}\right),\left|F_{\theta}^{\prime}(x)\right|$ can be bounded from below by estimating the variation on the angle of reflection after collision with the left wall. That variation can still be written as $\left|F_{\theta}^{\prime}(x)\right|=2|k(p(x, \theta))|\left|L^{\prime}(x)\right|$, where $L(x)$ is now the length of the segment of left wall from the left corner to the point of first collision. Due to the downward bending of the wall, it holds that $\left|L^{\prime}(x)\right| \geq\left|L^{\prime}(0)\right|=1$. Therefore $\left|F_{\theta}^{\prime}(x)\right|^{-1} \leq C$ for some $C>0$ which depends only on the curvature of the wall.

THEOREM 6.8. Under Assumptions 1, 2 and 3, the Markov operator of a random billiard is a Hilbert-Schmidt operator. In particular, its spectrum consists of eigenvalues $\lambda_{i} \in[-1,1]$, each of finite multiplicity, with 0 as the only accumulation point. Further, 1 is a simple eigenvalue.

PROOF. This is now a corollary of the previous lemma. Observe that the random billiard system is ergodic under these assumptions, so that 1 has multiplicity one.

It would be interesting to try to extract from this proof an estimate of the spectral gap of the Markov operator, something which we do not do here. In any event, it is worth making the following remarks. We recall that $P$ is said to have spectral gap $\gamma$ if there is $\rho=1-\gamma<1$ such that, for $\eta \in H$ satisfying $\eta(V)=0$ (that is, such that $\langle\eta, \mu\rangle=0$ ), the inequality

$$
\|P \eta\|_{2} \leq \rho\|\eta\|_{2}
$$

holds. In other words, $(\rho, 1)$ is the largest interval in $[-1,1]$, having 1 as a boundary point, that does not intersect the spectrum of $P$.

A large spectral gap implies fast convergence to the stationary measure $\mu$, in the following sense: for each $\eta \in H$ (the Hilbert space of signed measures on $V$ with square integrable densities with respect to $\mu$ ) such that $\eta(V)=0$, there is $C_{\eta}$ such that

$$
\left\|P^{k} \eta-\mu\right\|_{2} \leq C_{\eta} \rho^{k}
$$

for all $n \in \mathbb{N}$. 
It is possible to express $\gamma$ in terms of the random billiard map as follows. Define the Dirichlet form

$$
\mathscr{E}(\eta, \eta)_{\mu}:=\langle(I-P) \eta, \eta\rangle .
$$

Note that, for any constant $c$,

$$
\mathscr{E}(\eta-c \mu, \eta-c \mu)=\mathscr{E}(\eta, \eta),
$$

since $\mu$ is in the kernel of the self-adjoint operator $I-P$. A simple calculation shows that if $d \eta=\phi d \mu$ and $\Phi=\phi \circ \pi_{2}$, then

$$
\begin{aligned}
\mathscr{E}(\eta, \eta)_{\mu} & =\int_{E}(\Phi-\Phi \circ T) \Phi d v=\frac{1}{2} \int_{E}(\Phi-\Phi \circ T)^{2} d v \\
& =\frac{1}{2} \int_{X} \int_{V}\left[\phi(v)-\phi\left(\Psi_{x}(v)\right)\right]^{2} d \mu(v) d \lambda(x) .
\end{aligned}
$$

The spectral gap of the random billiard is now given by

$$
\begin{aligned}
\gamma & =\inf \left\{\frac{\mathscr{E}(\eta, \eta)_{\mu}}{\|\eta\|_{2}^{2}}: \eta(V)=0\right\} \\
& =\inf \left\{\frac{\int_{E}\left[\phi(v)-\phi\left(\Psi_{x}(v)\right)\right]^{2} d v}{2 \int_{V} \phi^{2} d \mu}: \phi \in L^{2}(V, \mu), \int_{V} \phi d \mu=0\right\} .
\end{aligned}
$$

\section{Diffusion limit}

From a random billiard system we obtain a random flight in a channel, as explained in Section 2.4, and to this random flight is associated a time-of-escape function $f(x)$, defined in the introduction. We expect $f(x)$ to contain information about the channel surface microgeometry. We have already seen in the introduction how such geometric information can be extracted from $f(x)$ in a simple example. In that case, the mean square displacement of a billiard particle along the axis of the channel is finite and the standard central limit theorem for Markov chains can be used to obtain the dependence of the diffusion constant

$$
D=\lim _{x \rightarrow \infty} \frac{x^{2}}{f(x)}
$$

on the ratio $b / h$, which is the sole geometric parameter of the family of Figure 2 . Before showing further details of this and indicating how something similar can be accomplished for more general geometries, it is perhaps useful to try to describe intuitively how the microgeometry can affect the diffusion constant $D$ by means of the following random walk model. 
7.1. A coin model of random flight. Fix a number $\alpha$ between 0 and 1 , which will be referred to as the surface slippage, and consider the random walk on a one-dimensional lattice with spacing $\delta$ between neighbor cells defined as follows. A point particle moves by jumping one step of length $\delta$ either backward or forward, the time between jumps being given by a constant $\bar{t}$. The direction of jump is decided by the rule that at each step we flip two coins simultaneously, one biased, with probability for heads equal to $\alpha$, and the other unbiased. If the biased coin comes up heads the particle repeats the behavior of the previous jump regardless of the outcome of the unbiased coin. We say in this case that it slips with probability $\alpha$. If the biased coin comes tails the particle forgets what it did in the previous jump and moves forward or backward depending on the outcome of the unbiased coin (say, heads = forward, tails = backward.)

There are only two directions of motion, represented by \pm 1 . The scattering operator $P$ for this example is the transition probabilities matrix

$$
P=\left(\begin{array}{cc}
\frac{1+\alpha}{2} & \frac{1-\alpha}{2} \\
\frac{1-\alpha}{2} & \frac{1+\alpha}{2}
\end{array}\right)
$$

The top left entry, $(1+\alpha) / 2$, is the probability that the particle direction in the next move is +1 given that it was +1 in the last move; the lower-left entry, $(1-\alpha) / 2$, is the probability that the next direction is +1 given that in the last move it was -1 , etc. Zero slippage corresponds to ordinary random walk on a one-dimensional lattice, in which no memory of past moves is kept. The case $\alpha=1$ corresponds to purely deterministic uniform motion forward or backward.

The Markov chain specified by $P$ has the same unique stationary distribution of directions for all $\alpha \neq 1$, namely, probability $1 / 2$ for both +1 and -1 . The limit diffusion process, however, has different diffusion constants depending on $\alpha$. More precisely, let $D_{\alpha}$ denote the constant for the process given as a limit of this random walk, by making $\delta$ and $\bar{t}$ go to 0 so that the ratio $D_{0}=\delta^{2} / \bar{t}$ remains constant. Then it can be shown, using the central limit theorem for Markov chains (stated in the next section), that

$$
D_{\alpha}=\frac{1+\alpha}{1-\alpha} D_{0}
$$

The reference diffusivity, $D_{0}$, in that of standard random walk.

It is worth emphasizing the following property of this example: the stationary distribution of directions is the same for all $\alpha$, but the speed of convergence to the stationary distribution varies greatly with slippage. In fact, the exponential rate of convergence is proportional to $1-\alpha$. Therefore, as $\alpha$ is close to 1 (high slippage), it will take longer to reach stationarity. The diffusivity constant will be 


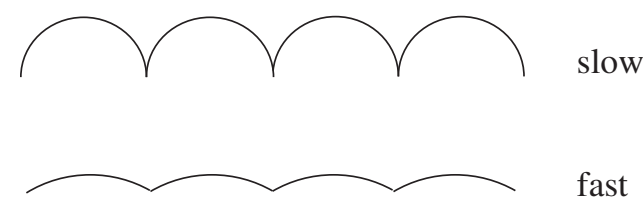

Figure 20. The top microgeometry produces relatively slow diffusion compared to the one at the bottom.

proportionally larger as particles effectively move a greater distance on average during a small interval of time.

We can draw a parallel between this example and the random flight for a random billiard system with microgeometries such as those of Figure 20. Although we do not yet have precise estimates, it can be seen numerically that the rate of convergence to the stationary distribution is faster for the top microgeometry, so that it can be assigned an effective value of $\alpha$ smaller than for the lower microgeometry. Numerical simulation of the random flight indicates, in fact, that the diffusion constant for the "flatter" microgeometry has larger diffusion constant. The example of Section 7.3 suggests that an effective measure of slippage $\alpha$ can be defined for random billiards as $1-\gamma$, where $\gamma$ is the spectral gap of $P$ (which is $1-\lambda$, where $\lambda$ is the second largest eigenvalue of $P$ after 1.)

7.2. Random flight with finite mean free path. Brownian motion on the real line has the following property: let $B_{t}$ denote the position of a particle undergoing standard Brownian motion starting at the origin at time $t=0$ and denote by $f(x)$ the mean time it takes $B_{t}$ to reach a distance $x$ from 0 . Then it is well-known that $D=x^{2} / 2 f(x)$, where $D=\sigma^{2} / 2$ and $\sigma^{2}$ is the variance of $B_{1}$. If a discrete time process has finite mean square jumps and satisfies the central limit theorem, then a limit diffusion is obtained with constant given by the limit of $x^{2} / 2 f(x)$ as $x \rightarrow \infty$. Thus the key issue we face is to find a central limit theorem that applies to the random flight of a random billiard system. Ideally, such a theorem will allow to relate $D$ to the spectrum of $P$.

The random displacement $X_{i}$ between two consecutive collisions with the channel wall has infinite mean square with respect to the stationary measure $\mu$ whenever $\mu$ is ergodic. Nevertheless, there are examples where this mean square displacement is finite, so that the considerations of the previous paragraph are valid. In such cases Theorem 7.1 can be used. The theorem applies to examples such as the 2 and 3-dimensional rectangular cells of the introduction and Section 3.1, as well as other rational polygonal billiard cells. This is an adaptation of a result due to Kipnis and Varadhan, [KiVa]. A similar theorem that applies to the more general $\mu$-ergodic case will be studied in a future paper.

We consider a particle undergoing random flight in a two or three-dimensional channel of infinite length and radius $r$. Let $V$ be, as before, the set of directions 
of the particle at the open side of a billiard cell and write $\Omega=V^{\mathbb{N}}$, with projections $\pi_{k}: \Omega \rightarrow V$. The particle has constant scalar velocity, $v$. We fix reference values $r_{0}$ and $v_{0}$ and write $r=r_{0} / \zeta, v=g(\zeta) v_{0}$, where $g(\zeta)$, until further specified, is an unbounded monotone increasing function. (It is mostly the case here that $g(\zeta)=\zeta$, but it will be convenient for later to define it more generally at this point.) The position of the particle along the axis of the channel at time $t$ is represented by $X_{\zeta, t}^{*}(\omega) \in \mathbb{R}$, for any $\omega \in \Omega$. We wish to study the limit of $X_{\zeta, t}^{*}$ as $\zeta \rightarrow \infty$.

Let $P$ be a bounded self-adjoint operator on $L^{2}\left(V, \mu^{\prime}\right)$, for some probability measure $\mu^{\prime}$, with spectrum in $[-1,1]$. Let $\Pi$ denote the spectral measure of $P$ and define, for a given $h \in L^{2}\left(V, \mu^{\prime}\right)$, the measure on $[-1,1]$ given by

$$
\varepsilon_{h}(d \lambda):=\langle h, \Pi(d \lambda) h\rangle .
$$

THEOREM 7.1. Give $\Omega$ the probability measure associated to $\left(P, \mu^{\prime}\right)$, where $P$ is the Markov operator for a choice of random billiard and $\mu^{\prime}$ is an ergodic stationary measure. Let $h(u)=2 r_{0} u \cdot e / u \cdot n,|u|=1$. Suppose that $h$ is square integrable with respect to $\mu^{\prime}$, has zero mean, and that

$$
\delta_{0}^{2}:=\int_{-1}^{1} \frac{1+\lambda}{1-\lambda} \varepsilon_{h}(d \lambda)<\infty .
$$

Also suppose that $g(\zeta)=\zeta$. Then, for any sequence $\zeta_{n} \rightarrow \infty$, the process $X_{\zeta_{n}, t}^{*}$ converges to Brownian motion with variance $\left(\delta_{0}^{2} / \tau_{0}\right) t$, where $\tau_{0}$ is the mean value of $2 r_{0} /\left(v_{0} u \cdot n\right)$ with respect to $\mu^{\prime}$. If $\left(P, \mu^{\prime}\right)$ is replaced with $\left(P, \delta_{\theta}\right)$, where the initial probability distribution is a delta-measure concentrated at an angle $\theta$ in the support of $\mu^{\prime}$, then the distribution of $X_{\zeta_{n}, t}^{*}$ will again converge to Brownian motion (with same variance), the convergence now being in measure as functions of $\theta$, relative to $\mu^{\prime}$.

As already noted, up to minor modifications having to do with our need to consider random times, Theorem 7.1 is due to Kipnis and Varadhan, [KiVa]. Clearly, requiring $h$ to be square integrable with respect to $\mu^{\prime}$ is very restrictive since it excludes all systems for which $\mu^{\prime}=\mu$. It is an open question whether there are any examples of billiard cells with square integrable $h$ that are not rational polygons. We apply below Theorem 7.1 to a couple of simple examples.

2-D boxes. This is the example of the introduction (Figure 2). For an initial $\theta_{0} \in(0, \pi)$, the possible states are $\theta_{0}$ and $\pi-\theta_{0}$, with transition probabilities shown on the Markov diagram of Figure 2. The transition probabilities matrix,

$$
P=\left(\begin{array}{cc}
p & 1-p \\
1-p & p
\end{array}\right)
$$


has eigenvalues 1 and $\lambda=2 p-1$, where $p$ depends on the geometric parameters as described in the introduction. The stationary probability distribution is $(1 / 2,1 / 2)$.

The function $h(\theta)$ can take values $\pm 2 r_{0} \cot \theta_{0}$, and $\varepsilon_{h}$ is the measure supported on $\lambda$, so that $\delta_{0}^{2}=\left(2 r_{0} \cot \theta_{0}\right)^{2}(1+\lambda) /(1-\lambda)$. The mean time between collisions is $\tau_{0}=2 r_{0} /\left(v_{0} \sin \theta_{0}\right)$. Then Theorem 7.1 gives that the diffusion limit is $\sigma B_{t}$, where $B_{t}$ is normalized Brownian motion (variance 1) and $\sigma^{2}=2 r_{0} v_{0} p(1-p)^{-1} \cos ^{2} \theta_{0} / \sin \theta_{0}$.

As a special case, suppose that $\theta_{0}=\pi / 4$, and that $b>2 h$. Then $k=0$, $s=2 h / b$, and $p=1-s$, so that

$$
\sigma^{2}=\sqrt{2} r_{0} v_{0}\left(\frac{b}{2 h}-1\right) .
$$

The interpretation of this result in terms of gas kinetics is that, if a pulse of noninteracting particles are released inside the channel with the same angle $\theta_{0}$, the transport along the channel can be approximated by the diffusion equation

$$
\frac{\partial u}{\partial t}=\frac{1}{2} \sigma^{2} \frac{\partial^{2} u}{\partial x^{2}},
$$

where $u(x, t)$ is the particle linear density, $t$ is time and $x$ is the coordinate along the axis of the channel.

3-D boxes. We consider now the example of Section 3.1. We refer to notation defined there. For any given initial direction, the function $h(v)$ can assume only four values: $\pm \delta_{1}, \pm \delta_{2}$. The spectral decomposition of the function $h$ is

$$
\left(\begin{array}{r}
\delta_{1} \\
\delta_{2} \\
-\delta_{2} \\
-\delta_{1}
\end{array}\right)=\frac{\delta_{1}+\delta_{2}}{2}\left(\begin{array}{r}
1 \\
1 \\
-1 \\
-1
\end{array}\right)+\frac{\delta_{1}-\delta_{2}}{2}\left(\begin{array}{r}
1 \\
-1 \\
1 \\
-1
\end{array}\right) \text {, }
$$

where $(1 / 2)(1,1,-1,-1)^{t}$ and $(1 / 2)(1,-1,1,-1)^{t}$ are associated to eigenvalues $\lambda_{2}$ and $\lambda_{3}$, respectively. The stationary measure is given by the probability distribution $(1 / 4,1 / 4,1 / 4,1 / 4)^{t}$ and the mean time between collisions is $\tau_{0}=$ $\left(\tau_{1}+\tau_{2}\right) / 2$. We have $\left(1+\lambda_{2}\right) /\left(1-\lambda_{2}\right)=p_{1} /\left(1-p_{1}\right)$ and $\left(1+\lambda_{3}\right) /\left(1-\lambda_{3}\right)=$ $p_{2} /\left(1-p_{2}\right)$. The diffusion limit in this case is Brownian motion with variance $\sigma^{2}$ given by

$$
\sigma^{2}=\left[\frac{p_{1}}{1-p_{1}}\left(\delta_{1}+\delta_{2}\right)^{2}+\frac{p_{2}}{1-p_{2}}\left(\delta_{1}-\delta_{2}\right)^{2}\right] \frac{1}{\tau_{0}} .
$$

The geometric parameters of the microgeometry are contained in the expressions for $p_{i}, \delta_{i}$ and $\tau_{i}$ given in Section 3.1. 
7.3. The ideal cavity model. The passage from the random flight to the diffusion process is in most cases not entirely standard if under the stationary distribution $\mu$ the mean square displacement is infinite. Nevertheless a central limit theorem still holds under appropriate normalization. More precisely, one needs here a central limit theorem that gives limit in distribution for sums of the form $\left(X_{1}+\cdots+X_{n}\right) / \sqrt{n \ln n}$ and expresses the variance of the limit random variable as a function of the spectrum of the Markov operator $P$. This will be discussed in a future paper. Here we only consider a single but representative example.

Nonstandard central limit theorems of this kind are used in a number of other studies. Most closely related to our concerns is [BGT]. The example discussed later, which is the Markov process defined by $P_{\text {Maxwell }}$ (see Section 3.2) is essentially the system studied in that paper. A central limit theorem similar to what we need is used in [Bl] and [SV], where the Lorentz gas model with infinite horizon is investigated See also [AD] and [Go].

We consider now the example of Section 3.2, given by $P_{\mu}+\alpha P_{\mu^{\perp}}$, where $P_{\mu}$ is the orthogonal projection to the 1-dimensional subspace spanned by the stationary measure $\mu$ and $P_{\mu \perp}$ is orthogonal projection to the orthogonal complement. Recall that $\alpha$ is interpreted as the probability that a particle bounces off the flat side in a specular way, and $1-\alpha$ the probability that it falls into the cavity and eventually exits with a probability distribution of angles given by $\mu$.

Theorem 7.1 does not apply here since the function $h(\theta)=2 r_{0} \cot \theta$ has infinite variance with respect to $\mu$. It turns out, however, that a diffusion limit can be obtained after appropriate scaling of the particle velocity. More precisely, the function $g(\zeta)$ that is required for the limit of $X_{\zeta, t}^{*}$ to be normally distributed is $g(\zeta)=\zeta / \ln \zeta$.

Proposition 7.2. Let $X_{\zeta, t}^{*}$ be as defined previously, now corresponding to the Markov operator $P=P_{\mu}+\alpha P_{\mu^{\perp}}$. Then for any $\zeta_{n} \rightarrow \infty$ and any fixed $t>0$, $X_{\zeta_{n}, t}^{*}$ converges in distribution to normal distribution with variance $t \sigma^{2}$, where

$$
\sigma^{2}=C \frac{1+\alpha}{1-\alpha}
$$

where the constant $C$ only depends on $r_{0}$ and $v_{0}$.

See [BGT] for details. Although the context of that paper is different from ours, their proof carries on to this case, with only minor modifications.

The ideal cavity model does not correspond, strictly speaking, to any microgeometry, but it is useful for intuitively understanding actual random billiard systems. Observe, for example, the two geometries of Figure 20. They can be compared with the ideal cavity by assigning a larger $\alpha$ for the one with smaller curvature. 


\section{Remark about residence time in cells}

The random walk on the line, driven by a random billiard map, only depends on the Markov operator $P$. For the strict random billiard model and its diffusion limit studied so far, the time a particle spends inside a billiard cell is considered negligible. But if we wish to go from an infinitesimal billiard geometry, in the strict sense defined above, to a small, but finite, deterministic billiard system at a scale comparable to that of the channel radius, it becomes important to estimate how much time, on average, a particle spends in the billiard cell. The mean time spent inside billiard cells, which we refer to as the residence time of the random billiard, would then be a numerical factor to be taken into account when describing the diffusion limit. This mean time can be calculated exactly, for ergodic billiards, by standard ergodic theory arguments. We show below how this average depend on the shape of the billiard cell.

Consider an ordinary billiard system with piecewise smooth boundary of total length $l$, a distinguished boundary component consisting of a line segment of length $o$, and enclosed area $A$. The distinguished flat side is parametrized by $[0,1]$. Let $E=[0,1] \times[0, \pi]$, as before, denote the part of the phase space for that flat side and suppose that the billiard system is ergodic. For each $(x, \theta) \in E$, let $S(x, \theta)$ and $N(x, \theta)$ be, respectively, the time of first return and the number of collisions before returning to the distinguished side, counting the arrival at the side as one collision. Denote by $\bar{E}$ the whole unit phase space (i.e., with scalar velocity equal to 1$)$ and define for any $(p, v) \in \bar{E}$ the function $\tau(p, v)$ that gives the time duration of the free flight with velocity $v$ from $p$ to the point of next collision. The average values of $N, S$ are defined by

$$
\begin{aligned}
\langle N\rangle_{E} & :=\frac{1}{2} \int_{0}^{1} \int_{0}^{\pi} N(x, \theta) \sin \theta d \theta d x, \\
\langle S\rangle_{E} & :=\frac{1}{2} \int_{0}^{1} \int_{0}^{\pi} S(x, \theta) \sin \theta d \theta d x .
\end{aligned}
$$

Similarly, define the average of $\tau$ (over $\bar{E}$ ), by

$$
\langle\tau\rangle_{\bar{E}}:=\int_{\bar{E}} \tau(\xi) d \bar{v}(\xi)
$$

where $\bar{v}$ is the normalized Liouville measure on $\bar{E}$. Let $B: \bar{E} \rightarrow \bar{E}$ denote the billiard map for the billiard table that includes the top flat side. The first return map to $E$ will be written $T: E \rightarrow E$. The billiard flow will be denoted $\varphi_{t}$. Elements of $\bar{E}$ will be written $\xi=(x, v)$. Note that

$$
S(\xi)=\tau(\xi)+\tau\left(B(\xi)+\cdots+\tau\left(B^{N(\xi)-1}(\xi)\right) .\right.
$$


A standard application of Birkhoff's ergodic theorem gives the following answer to the question posed at the top of the section.

THEOREM 8.1. Suppose that the billiard cell has finite diameter and that the system is ergodic. Let $u$ be the scalar velocity of the billiard particle. Then

(1) $\langle N\rangle_{E}=l / o$;

(2) $\langle S\rangle_{E}=A \pi / o u$,

(3) $\langle\tau\rangle_{\bar{E}}=A \pi / l u$, and

(4) $\langle S\rangle_{E}=\langle N\rangle_{E}\langle\tau\rangle_{\bar{E}}$.

PROOF. We give a brief sketch of the proof, which for the most part is a standard argument. For each $\xi \in E$ and positive integer $l$, define

$$
\begin{aligned}
N^{l}(\xi) & :=N(\xi)+N(T(\xi))+\cdots+N\left(T^{l}(\xi)\right) ; \\
S^{l}(\xi) & :=S(\xi)+S(T(\xi))+\cdots+S\left(T^{l}(\xi)\right) .
\end{aligned}
$$

Then $N^{l}(\xi)$ is the total number of collisions with the table boundary during the period of $l$ returns to the distinguished (top) side, and $S^{l}(\xi)$ is the total time during the same period. It is immediate that $\lim _{l \rightarrow \infty} N^{l}(\xi) / l=\langle N\rangle_{E}$ and $\lim _{l \rightarrow \infty} S^{l}(\xi) / l=\langle S\rangle_{E}$. Therefore, for $v$-a.e. $\xi \in E$,

$$
\begin{aligned}
\langle N\rangle_{E}^{-1} & =\lim _{l \rightarrow \infty} \frac{l}{N^{l}(\xi)}=\lim _{l \rightarrow \infty} \frac{1}{N^{l}(\xi)} \sum_{i=0}^{N^{l}(\xi)} \chi_{E}\left(B^{i}(\xi)\right) \\
& =\bar{v}(E)=\frac{\text { length of distinguished side }}{\text { total perimeter of table boundary }} .
\end{aligned}
$$

This shows (1). To obtain (4), start with

$$
\sum_{k=0}^{N^{l}(\xi)} \tau\left(B^{k}(\xi)\right)=S(\xi)+S(T(\xi))+\cdots+S\left(T^{l-1}(\xi)\right)
$$

and average both sides over $E$, using $T$-invariance of the measure $v$. This gives

$$
\left\langle\sum_{k=0}^{N^{l}(\xi)} \tau\left(B^{k}(\xi)\right)\right\rangle_{E}=l\langle S\rangle_{E}
$$

Consequently,

$$
\langle S\rangle_{E}=\lim _{l \rightarrow \infty}\left\langle\left(\frac{N^{l}(\xi)}{l}\right)\left(\frac{1}{N^{l}(\xi)} \sum_{k=0}^{N^{l}(\xi)} \tau\left(B^{k}(\xi)\right)\right)\right\rangle_{E}=\langle N\rangle_{E}\langle\tau\rangle_{\bar{E}} .
$$




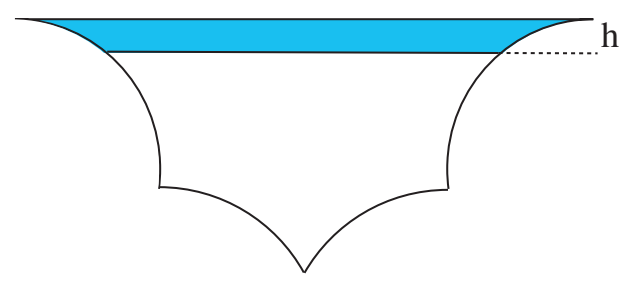

Figure 21. Define $U_{h}$ as the strip of width $h$ with the distinguished side as one of the boundary lines.

To show (2), it is convenient to first introduce the collar region $U_{h}$ shown in Figure 21, where $h$ is a small positive number.

Except for a set of small measure (which goes to zero with $h$ ), the time it takes for the ray with initial condition $\xi=(x, \theta) \in E$ to traverse $U_{h}$ is $\eta(\xi)=h / u \sin \theta$. An explicit integral calculation gives

$$
\lim _{h \rightarrow 0} \frac{1}{h}\langle\eta\rangle_{E}=\pi / 2 u \text {. }
$$

We can now conclude that, for $v$-a.e. $\xi \in E$,

$\langle S\rangle_{E} \frac{\text { length of top side }}{\text { area of billiard cell }}$

$$
\begin{aligned}
& =\lim _{h \rightarrow 0} \lim _{m \rightarrow \infty}\left(\frac{S^{m}(\xi)}{m}\right)\left(\frac{1}{h S^{m}(\xi)} \int_{0}^{S^{m}(\xi)} \chi_{U_{h}}\left(\varphi_{t}(\xi)\right)\right) d t \\
& =\lim _{h \rightarrow 0} \lim _{l \rightarrow \infty} \frac{1}{h m} \int_{0}^{S^{m}(\xi)} \chi_{U_{h}}\left(\varphi_{t}(\xi)\right) d t \\
& =\lim _{h \rightarrow 0} \lim _{l \rightarrow \infty} \frac{1}{h m} \sum_{i=0}^{m-1} 2 \eta\left(T^{i}(\xi)\right)=\lim _{h \rightarrow 0} \frac{2}{h}\langle\eta\rangle_{E}=\frac{\pi}{u} .
\end{aligned}
$$

This gives the average value of $S$ claimed in (2).

\section{References}

[AD] J. Aaronson and M. Denker. Local limit theorems for partial sums of stationary sequences generated by Gibbs-Markov maps, Stoch. Dyn. 1 (2001), 193-237.

[Ar] L. Arnold. Random dynamical systems, Springer, Berlin, 1998.

[ACM] G. Arya, H.-C. Chang, and E. J. Maginn. Knudsen diffusivity of a hard sphere in a rough slit pore, Phys. Rev. Lett. 91:2 (July 2003), 026102(4).

[Ba] H. Babovsky. On Knudsen flows within thin tubes, J. Stat. Phys. 44:5-6 (1986), 865-878. 
[BGT] C. Börgers, C. Greengard, and E. Thomann. The diffusion limit of free molecular flow in thin plane channels, SIAM J. Appl. Math. 52:4 (1992), 1057-1075.

[Bi] P. Billingsley. Convergence of probability measures, Wiley, New York, 1968.

[B1] P. M. Bleher. Statistical properties of two-dimensional periodic Lorentz gas with infinite horizon, J. of Stat. Physics 66:1 (1992), 315-373.

[Br] L. Breiman. Probability, Classics in Applied Mathematics 7, SIAM, Philadelphia, 1992.

[C1] C. Cercignani. The Boltzmann equation and its applications, Springer, New York, 1988.

[C2] C. Cercignani and D. H. Sattinger. Scaling limits and models in physical processes, DMV Seminar 28, Birkhäuser, Basel, 1998.

[CFS] I. P. Cornfeld, S. V. Fomin and Y. G. Sinai. Ergodic theory, Grundlehren der Mathematischen Wissenschaften (Fundamental Principles of Mathematical Sciences) 245, Springer, New York, 1982.

[FY1] R. Feres and G. Yablonsky. Knudsen's cosine law and random billiards, Chem. Eng. Sci. 59 (2004), 1541-1556.

[FY2] R. Feres and G. Yablonsky. Probing surface structure via time-of-escape analysis of gas in Knudsen regime, Chem. Eng. Sci. 61 (2006), 7864-7883.

[Go] S. Gouëzel. Central limit theorem and stable laws for intermittent maps, preprint, September, 2003.

[HK] B. Hasselblatt and A. Katok. Introduction to the modern theory of dynamical systems, Encyclopedia of Mathematics and its Applications 54, Cambridge University Press, Cambridge, 1995.

[KS] A. Katok and J.-M. Strelcyn. Invariant manifolds, entropy and billiards: Smooth maps with singularities, Lecture Notes in Mathematics 1222, Springer, Berlin, 1986.

[Ki] Y. Kifer. Ergodic theory of random transformations, Birkhäuser, Boston, 1986.

[KiVa] C. Kipnis and S. R. S. Varadhan. Central limit theorem for additive functionals of reversible Markov processes and applications to simple exclusions, Commun. Math. Phys. 104 (1986), 1-19.

[Kn] M. Knudsen. Kinetic theory of gases: Some modern aspects, Methuen's Monographs on Physical Subjects, Methuen, London, 1952.

[Re] D. Revuz. Markov chains, North-Holland, Amsterdam, 1975.

[SV] D. Szász and T. Varjú. Markov towers and stochastic properties of billiards, Modern dynamical systems and applications, M. Brin, B. Hasselblatt, Y. Pesin (eds.), 433-445, Cambridge University Press, Cambridge, 2004.

[Ta] S. Tabachnikov. Billiards, Panor. Synthèses 1, Société Mathématique de France, Paris, 1995.

[Wa] P. Walters. An introduction to ergodic theory, Springer, New York, 1982. 
RENATO FERES

WASHINGTON UNIVERSITY

DEPARTMENT OF MATHEMATICS

ST. LOUIS, MO 63130

UNITED STATES

feres@math.wustl.edu 